\title{
Parallel Nonoverlapping DDM Combined with the Characteristic Method for Incompressible Miscible Displacements in Porous Media
}

\author{
Keying Ma and Tongjun Sun \\ School of Mathematics, Shandong University, Jinan, Shandong 250100, China \\ Correspondence should be addressed to Keying Ma; makeying@sdu.edu.cn
}

Received 28 May 2012; Revised 27 October 2012; Accepted 1 November 2012

Academic Editor: J. Rappaz

Copyright (C) 2013 K. Ma and T. Sun. This is an open access article distributed under the Creative Commons Attribution License, which permits unrestricted use, distribution, and reproduction in any medium, provided the original work is properly cited.

\begin{abstract}
Two types of approximation schemes are established for incompressible miscible displacements in porous media. First, standard mixed finite element method is used to approximate the velocity and pressure. And then parallel non-overlapping domain decomposition methods combined with the characteristics method are presented for the concentration. These methods use the characteristic method to handle the material derivative term of the concentration equation in the subdomains and explicit flux calculations on the interdomain boundaries by integral mean method or extrapolation method to predict the inner-boundary conditions. Thus, the velocity and pressure can be approximated simultaneously, and the parallelism can be achieved for the concentration equation. The explicit nature of the flux prediction induces a time step limitation that is necessary to preserve stability. These schemes hold the advantages of nonoverlapping domain decomposition methods and the characteristic method. Optimal error estimates in $L^{2}$-norm are derived for these two schemes, respectively.
\end{abstract}

\section{Introduction}

The two-phase fluid displacements in porous media is one of the most important basic problems in the oil reservoir numerical simulation. It is governed by a nonlinear coupled system of partial differential equations with initial and boundary values. In this paper, we will consider the following incompressible miscible case: the pressure is governed by an elliptic equation and the concentration is governed by a convection-diffusion equation [1-5].

$$
-\nabla \cdot\left(\frac{k(x)}{\mu(c)}(\nabla p-\gamma(c) \nabla d(x))\right) \equiv \nabla \cdot \mathbf{u}=q, \quad x \in \Omega, t \in J,
$$

$$
\phi \frac{\partial c}{\partial t}+\mathbf{u} \cdot \nabla c-\nabla \cdot(D(\mathbf{u}) \nabla c)=(\widetilde{c}-c) \tilde{q}, \quad x \in \Omega, t \in J,
$$

$$
\begin{gathered}
\mathbf{u} \cdot v=(D(\mathbf{u}) \nabla c) \cdot v=0, \quad x \in \partial \Omega, t \in J, \\
c(x, 0)=c_{0}(x), \quad x \in \Omega,
\end{gathered}
$$

where $\Omega$ is a bounded domain in $\mathbf{R}^{2}, J=(0, T]$, and $\widetilde{q}=$ $\max \{q, 0\}$ is nonzero at injection wells only. The variables in (1a)-(1d) are the pressure $p(x, t)$ in the fluid mixture, the Darcy velocity $\mathbf{u}=\left(u_{1}, u_{2}\right)^{\prime}$, and the relative concentration $c(x, t)$ of the injected fluid. The $v$ is the unit outward normal vector on boundary $\partial \Omega$.

The coefficients and data in (1a)-(1d) are $k(x)$ : the permeability of the porous media; $\mu(c)$, the viscosity of the fluid mixture; $q(x, t)$ : representing flow rates at wells; $\gamma(c)$ and $d(x)$, the gravity coefficient and vertical coordinate; $\phi(x)$ : the porosity of the rock; $\widetilde{c}(x, t)$, the injected concentration at injection wells $(q>0)$ and the resident concentration at production wells $(q<0)$. Here, $D(\mathbf{u})$ is a tensor $2 \times 2$ matrix and generally has the form

$$
D(\mathbf{u})=\phi(x)\left\{d_{m} I+|\mathbf{u}|\left(d_{l} E(\mathbf{u})+d_{t} E^{\perp}(\mathbf{u})\right)\right\},
$$


where $2 \times 2$ matrix $E=\left(e_{i j}\right)$ satisfies $e_{i j}=u_{i} u_{j} /|\mathbf{u}|^{2}$, $E^{\perp}=I-E, d_{m}$ is the molecular diffusivity, and $d_{l}, d_{t}$ are longitudinal and transverse dispersivities, respectively. Furthermore, a compatibility condition $\int_{\Omega} q(x, t) d x=0$ must be imposed to determine the pressure.

The pressure equation is elliptic and easily handled by standard mixed finite element method, which has been proven to be an effective numerical method for solving fluid problems. It has an advantage to approximate the unknown variable and its diffusive flux simultaneously. There are many research articles on this method [6-9]. The concentration equation is parabolic and normally convection dominated. It is well known that standard Galerkin scheme applied to the convection dominated problems does not work well and produces excessive numerical diffusion or nonphysical oscillation. A variety of numerical techniques have been introduced to obtain better approximations for (1a)-(1d), such as characteristic finite difference method [10], characteristic finite element method [11], the modified of characteristic finite element method (MMOC-Galerkin) [12], and the Eulerian-Lagrangian localized adjoint method (ELLAM) [13].

It is well known that parallel algorithms, based upon overlapping or non-overlapping domain decompositions, are effective ways to solve the large scale of PDE systems for most practical problems in engineering (e.g., see [14-17]). We have presented parallel Galerkin domain decomposition procedures for parabolic equation [18-20]. These procedures use implicit Galerkin methods in the subdomains and explicit flux calculations on the interdomain boundaries by integral mean method or extrapolation-integral mean method. Some constraints for time step are still needed for these procedures to preserve stability, but less severe than that for fully explicit methods. With respect to the accuracy order of $h, L^{2}$-norm error estimates are optimal for higher-order finite element spaces and almost optimal for linear finite element space in twodimensional domain. Compared with Dawson-Dupont's schemes [21], these $L^{2}$-norm error estimates avoid the loss of $H^{-1 / 2}$ factor.

We also have considered using the procedures in [18] for wave equation [22] and convection-diffusion equation [23]. This paper is one of our sequent research papers. The main purpose is to use parallel Galerkin domain decomposition procedures in [18] combined with the characteristic method for the concentration equation of incompressible miscible displacements in porous media. This paper is organized as follows. In Section 2, we first present mixed finite element method for the velocity and pressure and then formulate parallel non-overlapping domain decomposition methods combined with the characteristics method for the concentration. We establish the combined approximation schemes. Auxiliary lemmas are listed in Section 3, which show some properties of finite element spaces and projections. In Section 4 , we derive the optimal-order $L^{2}$-norm error estimates. Finally in Section 5, we extend the consideration for another approximate scheme by using extrapolation method. It is worthwhile to specially emphasize that the research of this paper is creative. No former researchers discussed it. These schemes not only hold the advantages of non-overlapping domain decomposition methods, but also hold the advantages of the characteristic method.

\section{Formulation of the Methods}

In this paper, we adopt notations and norms of usual Sobolev spaces [3]:

$$
\begin{gathered}
H^{m}(\Omega)=\left\{f: \frac{\partial^{|\alpha|} f}{\partial x^{\alpha}} \in L^{2}(\Omega) \text { for }|\alpha| \leq m\right\}, \\
\|f\|_{m}^{2}=\sum_{|\alpha| \leq m}\left\|\frac{\partial^{|\alpha|} f}{\partial x^{\alpha}}\right\|^{2}, \quad m \geq 0, \\
W_{\infty}^{m}(\Omega)=\left\{f: \frac{\partial^{|\alpha|} f}{\partial x^{\alpha}} \in L^{\infty}(\Omega) \text { for }|\alpha| \leq m\right\}, \\
\|f\|_{W_{\infty}^{m}}=\max _{|\alpha| \leq m}\left\|\frac{\partial^{|\alpha|} f}{\partial x^{\alpha}}\right\|_{\infty}, \quad m \geq 0 .
\end{gathered}
$$

In particular, $H^{0}(\Omega)$ and $W_{\infty}^{0}(\Omega)=L^{\infty}(\Omega)$. The inner product on $L^{2}(\Omega)$ is denoted by $(\cdot, \cdot)$.

We also use the following spaces that incorporate time dependence. Let $[a, b] \subset J$ and let $X$ be any of the spaces just defined. For $f(x, t)$ suitably smooth on $\Omega \times[a, b]$, we let

$$
\begin{gathered}
H^{m}(a, b ; X)=\left\{f: \int_{a}^{b}\left\|\frac{\partial^{\alpha} f(\cdot, t)}{\partial t^{\alpha}}\right\|_{X} d t<\infty, \text { for } \alpha \leq m\right\}, \\
\|f\|_{H^{m}(a, b ; X)}=\left(\sum_{\alpha=0}^{m} \int_{a}^{b}\left\|\frac{\partial^{\alpha} f(\cdot, t)}{\partial t^{\alpha}}\right\|_{X} d t\right)^{1 / 2}, \quad m \geq 0 .
\end{gathered}
$$

Similarly, $W_{\infty}^{m}(a, b ; X)$ and the norm $\|f\|_{W_{\infty}^{m}(a, b ; X)}$ are defined. If $[a, b]=\bar{J}$, we simplify our notation and write $L^{\infty}\left(W_{\infty}^{1}\right)$ for $L^{\infty}\left(0, T ; W_{\infty}^{1}(\Omega)\right)$, and so forth.

If $f=\left(f_{1}, f_{2}\right)$ is a vector function, we note that $f \in X$ if $f_{1} \in X$ and $f_{2} \in X$. We also use the vector-function spaces and norms:

$$
\begin{gathered}
H^{m}(\operatorname{div} ; \Omega)=\left\{f: f_{1}, f_{2}, \nabla \cdot f \in H^{m}(\Omega)\right\}, \\
\|f\|_{H^{m}(\operatorname{div} ; \Omega)}^{2}=\left\|f_{1}\right\|_{m}^{2}+\left\|f_{2}\right\|_{m}^{2}+\|\nabla \cdot f\|_{m}^{2}, \quad m \geq 0, \\
H(\operatorname{div} ; \Omega)=H^{0}(\operatorname{div} ; \Omega) .
\end{gathered}
$$

We need some assumptions. The regularity assumptions on the solution of (1a)-(1d) are noted by [4]:

$$
(P):\left\{\begin{array}{l}
c \in L^{\infty}\left(H^{2}\right) \cap H^{1}\left(H^{1}\right) \cap L^{\infty}\left(W_{\infty}^{1}\right) \cap H^{2}\left(L^{2}\right), \\
p \in L^{\infty}\left(H^{1}\right), \\
\mathbf{u} \in L^{\infty}\left(H^{1}(\operatorname{div})\right) \cap L^{\infty}\left(W_{\infty}^{1}\right) \cap W_{\infty}^{1}\left(L^{\infty}\right) \cap H^{2}\left(L^{2}\right) .
\end{array}\right.
$$


We also require the following assumptions on the coefficients in (1a)-(1d) [4]. Let $a_{0}, a_{1}, \phi_{0}, \phi_{1}, d_{0}, d_{1}, K_{1}$, and $K_{2}$ be positive constants such that

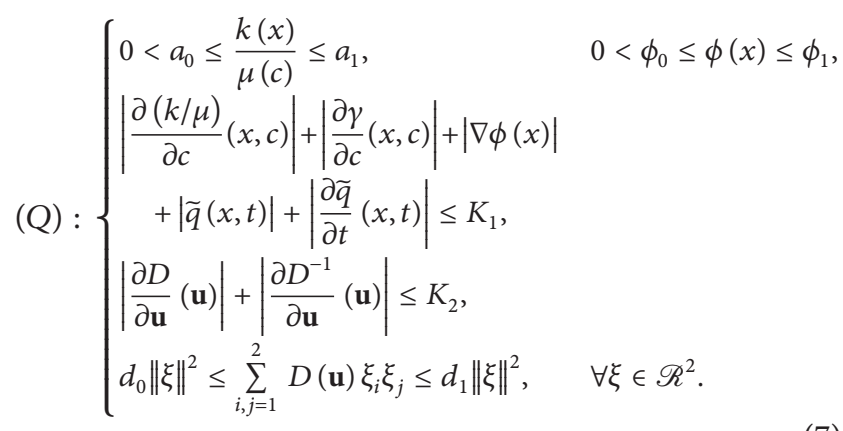

Further assumptions will be made in individual theorems as necessary.

For convenience, we assume that (1a)-(1d) is $\Omega$ periodic (see [4]); that is, all functions will be assumed to be spatially $\Omega$-periodic throughout the rest of this paper. This is physically reasonable, because no-flow conditions (1c) are generally treated by reflection, and in general, interior flow patterns are much more important than boundary effects in reservoir simulation. Thus, the boundary conditions (1c) can be dropped.

Throughout the analysis, $C$ and $K_{i}(i=3, \ldots, 6)$ will denote generic positive constants, independent of $h_{c}, h_{p}$, $\Delta t_{c}$, and $\Delta t_{p}$, but possibly depending on constants in $(Q)$, norms in $(P)$. Similarly, $\varepsilon$ will denote a generic small positive constant.

2.1. A Mixed Finite Element Method for the Pressure and Velocity. Let

$$
\begin{gathered}
H=\{\chi \in H(\operatorname{div} ; \Omega) \mid \chi \cdot v=0 \text { on } \partial \Omega\}, \\
W=\frac{L^{2}(\Omega)}{\{w \equiv \text { constant on } \Omega\}} .
\end{gathered}
$$

As in [4], the pressure equation (1a) is equal to the following saddle-point problem of finding a $\operatorname{map}(\mathbf{u}, p): J \rightarrow$ $H \times W$ such that

$$
\text { (a) } \begin{aligned}
A & (c ; \mathbf{u}, v)+B(v, p) \\
= & (\gamma(c) \nabla d, v), \quad \forall v \in H(\operatorname{div} ; \Omega),
\end{aligned}
$$

(b) $B(\mathbf{u}, w)=-(q, w), \quad \forall w \in L^{2}(\Omega)$,

where the bilinear forms

$$
\begin{gathered}
A(c ; \mathbf{u}, v)=\left(\frac{\mu(c)}{k(x)} \mathbf{u}, v\right), \\
B(v, p)=-(\nabla \cdot v, p), \\
B(\mathbf{u}, w)=-(\nabla \cdot \mathbf{u}, w) .
\end{gathered}
$$

For $h_{p}>0$, we discrete (10) in space on a quasiuniform mesh $\mathscr{T}_{h_{p}}$ of $\Omega$ with diameter of element $\leq h_{p}$. Let
$V_{h_{p}} \times W_{h_{p}} \subset H \times W$ be Raviart-Thomas spaces [6,7] of index $l=0$ for this mesh.

The mixed method for pressure and velocity, given a concentration approximation $C$ at a time $t \in J$, consists of $U \in V_{h_{p}}$ and $P \in W_{h_{p}}$ such that

$$
\begin{gathered}
A(C ; U, v)+B(v, P)=(\gamma(c) \nabla d, v), \quad \forall v \in V_{h_{p}}, \\
B(U, w)=-(q, w), \quad \forall w \in W_{h_{p}} .
\end{gathered}
$$

Existence and uniqueness of $U$ and $P$ are proved in [1], based on ideas of [24].

2.2. A Characteristic DDM for the Concentration. In this section, we assume $u$ in (1a)-(1d) is given. Define

$M=\left\{v \in L^{2}(\Omega) \mid v\right.$ is piecewise constant $\}$, $M$ is dense in $L^{2}(\Omega)$.

Let

$$
\begin{aligned}
\psi(x) & =\left[|\mathbf{u}(x)|^{2}+(\phi(x))^{2}\right]^{1 / 2} \\
& =\left[\left(u_{1}(x)\right)^{2}+\left(u_{2}(x)\right)^{2}+(\phi(x))^{2}\right]^{1 / 2},
\end{aligned}
$$

and let the characteristic direction associated with the operator $\phi \partial c / \partial t+\mathbf{u} \cdot \nabla c$ be denoted by $\tau$, where

$$
\psi(x) \frac{\partial c}{\partial \tau}=\phi(x) \frac{\partial c}{\partial t}+\mathbf{u} \cdot \nabla c .
$$

The weak form of (1a)-(1d) is finding a map $c: J \rightarrow$ $L^{2}(\Omega)$ such that

$$
\begin{aligned}
& \text { (a) }\left(\psi \frac{\partial c}{\partial \tau}, \varphi\right)+((D(\mathbf{u}) \nabla c), \nabla \varphi) \\
& =((\widetilde{c}-c) \tilde{q}, \varphi), \quad \forall \varphi \in L^{2}(\Omega), \\
& \text { (b) } c(x, 0)=c_{0}(x), \quad \forall x \in \Omega .
\end{aligned}
$$

Part $J$ into $0=t^{0}<t^{1}<\cdots<t^{N}=T$, with $\Delta t_{c}^{n}=t^{n}-t^{n-1}$. The analysis is valid for variable time steps, but we drop the superscript from $\Delta t_{c}$ for convenience. For functions $f$ on $\Omega \times J$, we write $f^{n}(x)$ for $f\left(x, t^{n}\right)$. Approximate $\left(\partial c^{n} / \partial \tau\right)(x)=$ $(\partial c / \partial \tau)\left(x, t^{n}\right)$ by a backward difference quotient in the $\tau$ direction,

$$
\frac{\partial c^{n}}{\partial \tau}(x) \simeq \frac{c^{n}(x)-c^{n-1}\left(x-(\mathbf{u}(x) / \phi(x)) \Delta t_{c}\right)}{\Delta t_{c} \sqrt{1+|\mathbf{u}(x)|^{2} /(\phi(x))^{2}}} .
$$

If we let $\bar{x}=x-(\mathbf{u}(x) / \phi(x)) \Delta t_{c}$ and $\bar{f}(x)=f(\bar{x})$, then we get

$$
\psi \frac{\partial c^{n}}{\partial \tau} \simeq \phi \frac{c^{n}-\bar{c}^{n-1}}{\Delta t_{c}} .
$$

Since the problem is $\Omega$-periodic [4], $\bar{c}^{n-1}$ is always defined and the tangent to the characteristic (i.e., the $\tau$ segment) 


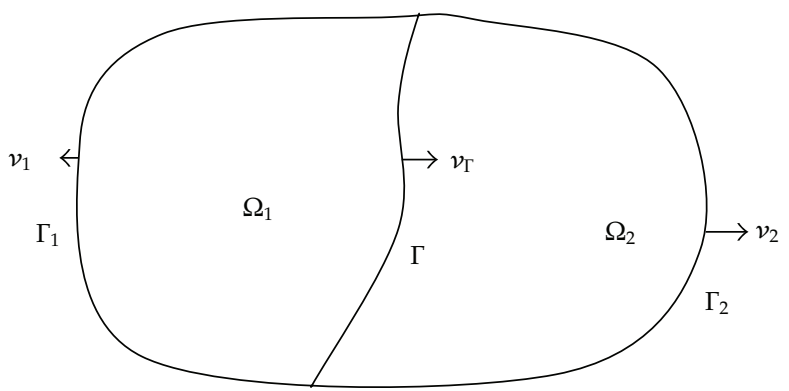

FIgURE 1: The domain $\Omega$ with the interdomain boundary $\Gamma$.

cannot cross a boundary to an undefined location. The difference quotient relates the concentration at a given $x$ at time $t^{n}$ to the concentration that would flow to $x$ from time $t^{n-1}$ if the problem were purely hyperbolic.

The time difference (18) will be combined with a characteristic DDM in the space variables. We recall the domain decomposition procedures in [18] here. For simplicity and without losing generality, we only discuss the case of two subdomains. But the algorithms and theories can be extended to the case of many subdomains. Divide $\Omega$ into two subdomains $\Omega_{j}(j=1,2)$ by an interdomain boundary $\Gamma$, which is a surface of dimension $d-1$, see Figure 1 . We denote by $\Gamma_{j}=\partial \Omega_{j} \bigcap \partial \Omega$ the part of the boundary of the subdomains which coincides with $\partial \Omega$. Denote the unit vector normal to $\Gamma$ as $\boldsymbol{v}_{\Gamma}$, which points from $\Omega_{1}$ toward $\Omega_{2}$.

Let $\mathscr{T}_{j, h_{c}}$ be quasi-uniform partitions of $\Omega_{j}(j=1,2)$ and $\mathscr{T}_{h_{c}}=\mathscr{T}_{1, h_{c}} \cup \mathscr{T}_{2, h_{c}}$. Here, $h$ denotes the maximal element diameter of $\mathscr{T}_{h_{c}}$. We construct the finite element space $\mathscr{M}_{h_{c}}$ on $\mathscr{T}_{h_{c}}$ which satisfies the following condition (I)

(1) For $j=1,2$, let $\mathscr{M}_{j, h_{c}}$ be a finite element subspace of $H^{1}\left(\Omega_{j}\right)$, and let $\mathscr{M}_{h_{\mathrm{c}}} \subset L^{2}(\Omega)$ such that if $v \in \mathscr{M}_{h_{\mathrm{c}}}$, then $\left.v\right|_{\Omega_{j}} \in \mathscr{M}_{j, h_{c}}$.

(2) For $j=1,2, P_{r}\left(\Omega_{j}\right) \subset \mathscr{M}_{j, h_{c}}$, where $P_{r}\left(\Omega_{j}\right)$ is a polynomial space of degree at most $r$.

(3) For $j=1,2, h \in(0,1]$, the integer $k \geq 1$, and $u \in H^{k}\left(\Omega_{j}\right)$, there exists a positive constant $C$ independent of $h$ such that

$$
\inf _{v \in \mathscr{M}_{j, h_{c}}}\|u-v\|_{H^{s}\left(\Omega_{j}\right)} \leq C h^{\sigma}\|u\|_{H^{k}\left(\Omega_{j}\right)}, \quad 0 \leq s \leq 1
$$

where $\sigma=\min (r+1-s, k-s)$.

From the definition above, we note that functions $v$ in $\mathscr{M}_{h_{\mathrm{c}}}$ have a well-defined jump $[v]$ on $\Gamma$ :

$$
[v](\mathbf{x})=v\left(\mathbf{x}^{+}\right)-v\left(\mathbf{x}^{-}\right), \quad \forall \mathbf{x} \text { on } \Gamma,
$$

where $v\left(\mathbf{x}^{ \pm}\right):=\lim _{\lambda \rightarrow 0^{ \pm}} v\left(\mathbf{x}+\lambda \boldsymbol{v}_{\Gamma}\right)$.

To construct parallel algorithm, for a small constant $0<$ $H<\min \left\{\operatorname{diam}\left(\Omega_{1}\right), \operatorname{diam}\left(\Omega_{2}\right)\right\}$, we introduce an integral mean value of a given function $V \in L^{2}(\Omega)$ on the interdomain boundary $\Gamma$ as

$$
\bar{V}_{H}(\mathbf{x})=\frac{1}{2 H} \int_{-H}^{H} V\left(\mathbf{x}+\lambda \boldsymbol{v}_{\Gamma}\right) d \lambda, \quad \forall \mathbf{x} \text { on } \Gamma .
$$

Furthermore, we define the extrapolation of $\bar{V}_{H}(\mathbf{x})$ on $\Gamma$ as

$$
\widehat{V}_{H}(\mathbf{x})=\frac{4 \bar{V}_{H / 2}(\mathbf{x})-\bar{V}_{H}(\mathbf{x})}{3}, \quad \forall \mathbf{x} \text { on } \Gamma \text {. }
$$

Generally, near the intersection of boundary $\partial \Omega$ and inner-boundary $\Gamma$, the value of $V$ outside $\Omega$ is needed for $\bar{V}_{H}$ and $\widehat{V}_{H}$. If $\mathbf{x} \notin \Omega$, let $\widetilde{\mathbf{x}} \in \Omega$ denote the symmetric point of $\mathbf{x}$ with respect to $\partial \Omega$. For a given function $u \in L^{2}(\Omega)$, we define

$$
E u(\mathbf{x})= \begin{cases}u(\mathbf{x}), & \text { if } \mathbf{x} \in \Omega, \\ u(\widetilde{\mathbf{x}}), & \text { if } \mathbf{x} \notin \Omega .\end{cases}
$$

By (23), we know $\bar{V}_{H}$ and $\widehat{V}_{H}$ have the values on a strip domain $G=\left\{\mathbf{y} \mid \mathbf{y}=\mathbf{x}+\lambda \boldsymbol{v}_{\Gamma}, \lambda \in[-H, H], \forall \mathbf{x}\right.$ on $\left.\Gamma\right\}$, see Figure 2.

Now, we present the non-overlapping characteristic DDM for the concentration equation:

$$
\begin{aligned}
& \left(\phi \frac{C^{n}-\widehat{C}^{n-1}}{\Delta t_{c}}, v\right)+\left(\mathbf{D} \nabla C^{n}, \nabla v\right)+\left(q^{n} C^{n}, v\right) \\
& \quad+\left(\bar{C}_{\mu, H}^{n-1},[v]\right)_{\Gamma}+\left(\bar{v}_{\mu, H},\left[C^{n-1}\right]\right)_{\Gamma} \\
& \quad+d_{1} K H^{-1}\left(\left[C^{n-1}\right],[v]\right)_{\Gamma}=\left(q^{n} \widetilde{c}^{n}, v\right), \quad \forall v \in \mathscr{M}_{h_{c}},
\end{aligned}
$$

where

$$
\begin{gathered}
\widehat{C}^{n-1}(x)=C^{n-1}\left(x-\frac{U^{n}(x)}{\phi(x)} \Delta t_{c}\right), \\
C_{\mu}^{n-1}=\left(\mathrm{D} \nabla E C^{n-1}\right) \cdot v_{\Gamma} .
\end{gathered}
$$

The coefficient $K$ will be given in Lemma 3 .

2.3. The Combined Approximate Schemes. We now present our sequential time-stepping procedure that combines (24) and (12). As in [4], let us part $J$ into pressure time steps $0=$ $t_{0}<t_{1}<\cdots<t_{M}=T$, with $\Delta t_{p}^{m}=t_{m}-t_{m-1}$. Each pressure step is also a concentration step; that is, for each $m$ there exists $n$ such that $t_{m}=t^{n}$; in general, $\Delta t_{p}>\Delta t_{c}$. We may vary $\Delta t_{p}$, but except for $\Delta t_{p}^{1}$ we drop the superscript. For functions $f$ on $\Omega \times J$, we write $f_{m}(x)$ for $f\left(x, t_{m}\right)$; thus, subscripts refer to pressure steps and superscripts to concentration steps. 


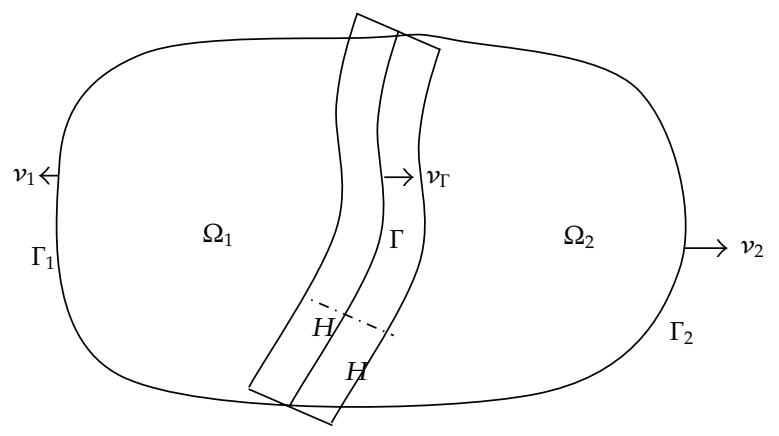

FIgure 2: The strip domain $G$ with width $2 H$.

If concentration step $t^{n}$ relates to pressure steps by $t_{m-1}<$ $t^{n} \leq t_{m}$, we require a velocity approximation for (25) based on $U_{m-1}$ and earlier values. If $m \geq 2$, take the linear extrapolation of $U_{m-1}$ and $U_{m-2}$ defined by

$$
F U^{n}=\left(1+\frac{t^{n}-t_{m-1}}{t_{m-1}-t_{m-2}}\right) U_{m-1}-\frac{t^{n}-t_{m-1}}{t_{m-1}-t_{m-2}} U_{m-2} .
$$

If $m=1$, set

$$
F U^{n}=U_{0}
$$

We retain the superscript on $\Delta t_{p}^{1}$ because $F U^{n}$ is first order correct in time during the first pressure step and second order during later steps.

The combined time-stepping procedure is finding a map $C:\left\{t^{0}, t^{1}, \ldots, t^{N}\right\} \rightarrow \mathscr{M}_{h_{c}}$ and a map $(U, P):$ $\left\{t_{0}, t_{1}, \ldots, t_{M}\right\} \rightarrow V_{h_{p}} \times W_{h_{p}}$ such that

$$
\begin{gathered}
C^{0}=\widetilde{C}^{0}, \\
A\left(C_{m} ; U_{m}, z\right)+B\left(z, P_{m}\right)=\left(\gamma\left(C_{m}\right) \nabla d, z\right), \quad \forall z \in V_{h_{p}}, \\
B(28 \mathrm{~b}) \\
\left(U_{m}, w\right)=-\left(q_{m}, w\right), \quad \forall w \in W_{h_{p}}, m \geq 0, \\
\left.+\frac{C^{n}-\check{C}^{n-1}}{\Delta t_{c}}, v\right)+\left(\mathbf{D} \nabla C^{n}, v\right)+\left(q^{n} C^{n}, v\right)+\left(\bar{C}_{\mu, H}^{n-1},[v]\right)_{\Gamma} \\
+\left(\bar{v}_{\mu, H},\left[C^{n-1}\right]\right)_{\Gamma}+d_{1} K H^{-1}\left(\left[C^{n-1}\right],[v]\right)_{\Gamma} \\
=\left(q^{n} \widetilde{c}^{n}, v\right), \quad \forall v \in \mathscr{M}_{h_{c}}, n \geq 1,
\end{gathered}
$$

where

$$
\check{C}^{n-1}(x)=C^{n-1}(\check{x})=C^{n-1}\left(x-\frac{F U^{n}(x)}{\phi(x)} \Delta t_{c}\right),
$$

$\widetilde{C}^{0}$ is given by (45).
The steps of the above calculation are as follows:

Step 1. $C^{0}$ known $\rightarrow$ solve $\left(U_{0}, P_{0}\right)$ by $(28 \mathrm{~b})$ and (28c); Step 2. On each domain, use (28d) to parallelly compute $\left\{C^{j}\right\}_{j=1}^{n_{1}}$ until $t^{n_{1}}=t_{1}$

Step 3. Then by (28b) and (28c) for $\left(U_{1}, P_{1}\right)$;

Step 4. Calculate the approximations in turn analogically to get the pressure, velocity, and concentration at other time-step, respectively.

The convergence analysis will make use of an analogue of $\widehat{x}$ defined for the exact velocity $\mathbf{u}^{n}$. If $f$ is a function on $\Omega$, set

$$
\check{f}(x)=f(\check{x})=f\left(x-\frac{F \mathbf{u}^{n}(x)}{\phi(x)} \Delta t_{c}\right) .
$$

The time step $t^{n}$ will be clear from the context.

Remark 1. In the scheme (28a)-(28d), the numerical flux on $\Gamma$ is computed explicitly from $C^{n-1}$, so that $C^{n}$ can be computed on $\Omega_{1}$ and $\Omega_{2}$ fully parallel once $C^{n-1}$ has been got.

\section{Auxiliary Lemmas}

We adopt some auxiliary lemmas about the finite element spaces, which will be used in the next section.

3.1. For the Pressure and Velocity. The Raviart-Thomas spaces $V_{h_{p}} \times W_{h_{p}}$ in Section 2.1 possess the following approximation and inverse properties [7]:

$$
\left(A_{p}\right):\left\{\begin{array}{l}
\inf _{v \in V_{h_{p}}}\|f-v\| \leq K_{3} h_{p}\|f\|_{1}, \\
\inf _{v \in V_{h_{p}}}\|f-v\|_{H(\text { div })} \leq K_{3} h_{p}\|f\|_{H^{1}(\text { div })}, \\
\inf _{w \in W_{h_{p}}}\|g-w\| \leq K_{3} h_{p}\|g\|_{1},
\end{array}\right.
$$

$$
\left(I_{p}\right): \begin{cases}\|v\|_{L^{\infty}} \leq K_{3} h_{p}^{-1}\|v\|, & \forall v \in V_{h_{p}}, \\ \|v\|_{W_{\infty}^{1}\left(T_{p}\right)} \leq K_{3} h_{p}^{-1}\|v\|_{L^{\infty}\left(T_{p}\right)}, & \forall v \in V_{h_{p}},\end{cases}
$$

where $K_{3}$ is a positive constant independent of $h_{p}$ and $T_{p}$ is an element of the mesh $\mathscr{T}_{h_{p}}$. 
Define the map $(\widetilde{U}, \widetilde{P}): J \rightarrow V_{h_{p}} \times W_{h_{p}}$ by (see [7])

$$
\begin{gathered}
A(c ; \widetilde{U}, v)+B(v, \widetilde{P})=(\gamma(c) \nabla d, v), \quad \forall v \in V_{h_{p}}, \\
B(\widetilde{U}, w)=-(q, w), \quad \forall w \in W_{h_{p}},
\end{gathered}
$$

where $c$ is the exact solution of (1a)-(1d).

By arguments in $[1,24]$, the map $(\widetilde{U}, \widetilde{P})$ exists and $\left(A_{p}\right)$ implies that

$$
\begin{aligned}
\| u-\widetilde{U} & \left\|_{L^{\infty}(H(\mathrm{div}))}+\right\| p-\widetilde{P} \|_{L^{\infty}\left(L^{2}\right)} \\
& \leq K_{4}\left\{\inf _{v \in V_{h_{p}}}\|u-v\|_{H(\text { div })}+\inf _{w \in W_{h_{p}}}\|p-w\|\right\} \\
& \leq K_{4}\left(\|u\|_{L^{\infty}\left(H^{1}(\text { div })\right)},\|p\|_{L^{\infty}\left(H^{1}\right)}\right) h_{p} .
\end{aligned}
$$

The positive constant $K_{4}$ depends on constant in (Q) but independent of $h_{p}, u, p$, and $c$.

The estimate (33) and $\left(I_{p}\right)$ imply that

$$
\|\widetilde{U}\|_{L^{\infty}\left(L^{\infty}\right)} \leq K_{5} .
$$

As in [1], comparison of (32) and (12) implies that

$$
\|U-\widetilde{U}\|_{H(\text { div })}+\|P-\widetilde{P}\| \leq K_{6}\left(1+\|\widetilde{U}\|_{L^{\infty}}\right)\|c-C\| .
$$

The estimates (33) and (35) will handle the coupling of concentration and velocity errors in the convergence analysis.

3.2. For the Concentration. In this section, we adopt some following lemmas [18].

Lemma 2. For smooth enough function $W$, there holds estimates:

$$
\begin{gathered}
\left\|\bar{W}_{H}-W\right\|_{L^{2}(\Gamma)} \leq \sqrt{2 H}\|\nabla W\|_{L^{2}(\Omega)}, \\
\left\|\bar{W}_{H}-W\right\|_{L^{\infty}(\Gamma)} \leq C H^{2}\|W\|_{W^{2, \infty}(\Omega)},
\end{gathered}
$$

$$
\begin{aligned}
W & (\mathbf{x})-\bar{W}_{H}(\mathbf{x}) \\
& =-\frac{1}{6} H^{2} W_{v_{\Gamma}^{2}}(\mathbf{x})-\frac{1}{120} H^{4} W_{v_{\Gamma}^{4}}(\mathbf{x})+o\left(H^{6}\right), \quad \forall \mathbf{x} \text { on } \Gamma,
\end{aligned}
$$

where $W_{v_{\Gamma}^{2}}$ and $W_{v_{\Gamma}^{4}}$ are the second and fourth normal derivative of $W$ on $\Gamma$, respectively.

Lemma 3. Let $G=\left\{\mathbf{y} \mid \mathbf{y}=\mathbf{x}+t \boldsymbol{v}_{\Gamma}, t \in[-H, H], \forall \mathbf{x}\right.$ on $\left.\Gamma\right\}$. If $\psi \in H^{1}(\Omega)$ and $H>0$ is small, one has

$$
\begin{gathered}
\|E \psi\|_{L^{2}(G)} \leq \sqrt{K}\|\psi\|_{L^{2}(\Omega)}, \\
\left\|\nabla(E \psi) \cdot v_{\Gamma}\right\|_{L^{2}(G)} \leq \sqrt{K}\|\nabla \psi\|_{L^{2}(\Omega)},
\end{gathered}
$$

where

$$
K= \begin{cases}1, & \text { if } G \subset \Omega, \\ 2, & \text { if } G \not \subset \Omega .\end{cases}
$$

Define an elliptic projection $\widetilde{\widetilde{C}} \in \mathscr{M}_{h_{c}}$ for $c: \forall v \in \mathscr{M}_{j, h_{c}}$, $j=1,2$,

$$
\begin{aligned}
(\mathbf{D} \nabla & \widetilde{\widetilde{C}}(t), \nabla v)+(q(t) \widetilde{\widetilde{C}}(t), v) \\
& =((\mathbf{D} \nabla c(t), \nabla v)+(q(t) c(t), v)) \\
& =\left(q(t) \overline{\bar{c}}(t)-\phi \frac{\partial c}{\partial t}(t)-\mathbf{u}(t) \cdot \nabla c(t), v\right)+(-1)^{j+1}(g, v)_{\Gamma},
\end{aligned}
$$

where $g=(\mathbf{D} \nabla c) \cdot \boldsymbol{v}_{\mathbf{\Gamma}}$. Let $\eta=c-\widetilde{\widetilde{C}}$. From [25-28], we see the following.

Lemma 4. For $\eta$ defined by (39), there holds the following. $L^{2}$-norm error estimate

$$
\begin{aligned}
\max _{0 \leq t \leq T}\|\eta\|_{L^{2}(\Omega)}+\left\|\eta_{t}\right\|_{L^{2}\left(0, T ; L^{2}(\Omega)\right)} & \\
& \leq C h_{c}^{r+1}\left\{\|u\|_{L^{\infty}\left(0, T ; H^{r+1}(\Omega)\right)}+\left\|u_{t}\right\|_{L^{2}\left(0, T ; H^{r+1}(\Omega)\right)}\right\},
\end{aligned}
$$

$L^{\infty}$-norm error estimate

$$
\begin{array}{r}
\|\eta\|_{L^{\infty}(\Omega)}+\left\|\eta_{t}\right\|_{L^{\infty}(\Omega)} \\
\leq C h_{c}^{2}\left|\ln h_{c}\right|\left\{\|u\|_{W^{2, \infty}(\Omega)}+\left\|u_{t}\right\|_{W^{2, \infty}(\Omega)}\right\}, \\
\text { if } r=1, d=2,
\end{array}
$$

$\|\eta\|_{L^{\infty}(\Omega)}+\left\|\eta_{t}\right\|_{L^{\infty}(\Omega)}$

$$
\leq C h_{c}^{r+1}\left\{\|u\|_{W^{r+1, \infty}(\Omega)}+\left\|u_{t}\right\|_{W^{r+1, \infty}(\Omega)}\right\}, \text { if } r>1 .
$$

For functions $\psi$ with restrictions in $H^{1}\left(\Omega_{1}\right) \cup H^{1}\left(\Omega_{2}\right)$, we define a norm

$$
\|\mid \psi\|^{2}=(D \nabla \psi, \nabla \psi)+d_{1} K H^{-1}([\psi],[\psi])_{\Gamma} .
$$

Lemma 5. There exists a positive constant $C_{0}=1-(\sqrt{2} / 2)$ such that for small $H>0$,

$$
\begin{aligned}
C_{0}\|\| \psi \|\left.\right|^{2} \leq & (\mathbf{D} \nabla \psi, \nabla \psi)+d_{1} K H^{-1}([\psi],[\psi])_{\Gamma} \\
& +2\left(\bar{\psi}_{\mu, H},[\psi]\right)_{\Gamma}, \quad \forall \psi \in \mathscr{M}_{h_{c}} .
\end{aligned}
$$

As we have shown, the combined approximation scheme (28d) includes two terms on the interdomain boundary $\Gamma$ by integral mean method to present explicit flux calculation. These terms are distinct ones different from DawsonDupont's schemes such that the standard elliptic projection (39) is insufficient for optimal error estimates. To get optimal error estimates, we need a new elliptic projection including terms on $\Gamma$. This new elliptic projection $\widetilde{C} \in \mathscr{M}_{h_{c}}$ of the solution $c$ is defined as

$$
\begin{gathered}
(\mathbf{D} \nabla(c-\widetilde{C}), \nabla v)+\left(\overline{(c-\widetilde{C})_{\mu, H}},[v]\right)_{\Gamma}+\left(\bar{v}_{\mu, H},[c-\widetilde{C}]\right)_{\Gamma} \\
\quad+d_{1} K H^{-1}([c-\widetilde{C}],[v])_{\Gamma}=0, \quad \forall v \in \mathscr{M}_{h_{c}} .
\end{gathered}
$$


It follows from Lemma 5 that the projection problem (44) has a unique solution for small $H$. Choose the initial $\widetilde{C}^{0}$ to be the projections of $c_{0}(x)$ by (44) and take the initial conditions

$$
C^{0}=\widetilde{C}^{0}
$$

Let

$$
\xi^{n}=c^{n}-\widetilde{C}^{n}, \quad \theta^{n}=C^{n}-\widetilde{C}^{n} .
$$

The following lemma gives the bounds of $\xi^{n}$.

Lemma 6. There holds a priori estimates:

$$
\begin{aligned}
\|\xi\|_{L^{2}(\Omega)} \leq C\left\{h_{c}^{r+1}+H^{1 / 2}\|\eta\|_{L^{\infty}(\Omega)}\right\}, \\
\left\|\xi_{t}\right\|_{L^{2}(\Omega)} \leq C\left\{h_{c}^{r+1}+H^{1 / 2}\left\|\eta_{t}\right\|_{L^{\infty}(\Omega)}\right\} .
\end{aligned}
$$

\section{A Priori Error Estimates in $L^{2}$-Norm}

Now, we turn to derive an optimal priori error estimate in $L^{2}$ norm for the concentration of approximation (28d). Optimal error estimates for velocity in $H(\operatorname{div} ; \Omega)$ and pressure in $L^{2}(\Omega)$ follow at once from (33) and (35).

It follows from trace theorem in [29] that

$$
\|\psi\|_{L^{2}(\Gamma)}^{2} \leq C_{2}\|\psi\|\|\psi\|_{1}, \quad \forall \psi \in H^{1}(\Omega)
$$

which will be used in the following proof. Then, we can derive an $L^{2}(\Omega)$-norm error estimate for $\theta^{n}$.

Lemma 7. For $\theta^{n}$ defined by (46), there holds the following error estimate:

$$
\begin{gathered}
\max _{0 \leq n \leq N}\left\|\theta^{n}\right\|^{2} \\
\leq C\left\{H \left[\max _{1 \leq n \leq N}\left(\left\|\eta^{n}\right\|_{L^{\infty}(\Omega)}^{2}+\left\|\eta^{n}\right\|^{2}\right)+\left\|\eta_{t}\right\|_{L^{2}\left(0, T ; L^{\infty}(\Omega)\right)}^{2}\right.\right. \\
\left.+\left\|\eta_{t}\right\|_{L^{2}\left(0, T ; L^{2}(\Omega)\right)}^{2}\right]+\left(\Delta t_{c}\right)^{2}+H^{5}+h_{p}^{2 l+2} \\
\left.+h_{c}^{2 r+2}+\left(\Delta t_{p}^{1}\right)^{3}+\left(\Delta t_{p}\right)^{4}\right\}
\end{gathered}
$$

provided

$$
\Delta t \leq C_{1} H^{2}, \quad C_{1}=\frac{d_{0}(1-\delta)^{2} C_{0}^{3}}{16 d_{1}^{2} K^{2} C_{2}^{2}}, \quad \forall 0<\delta \ll 1
$$

Proof. Combining (39) and (44), we have $\forall v \in \mathscr{M}$,

$$
\begin{gathered}
\left(\phi \frac{\widetilde{C}^{n}-\widetilde{C}^{n-1}}{\Delta t_{c}}, v\right)+\left(\mathbf{D} \nabla \widetilde{C}^{n}, \nabla v\right)+\left(\overline{\widetilde{C}}_{\mu, H}^{n-1},[v]\right)_{\Gamma} \\
+\left(\bar{v}_{\mu, H},\left[\widetilde{C}^{n-1}\right]\right)_{\Gamma}+d_{1} K H^{-1}\left(\left[\widetilde{C}^{n-1}\right],[v]\right)_{\Gamma}
\end{gathered}
$$

$$
\begin{aligned}
& =\left(\overline{\widetilde{C}}_{\mu, H}^{n-1}-\overline{\widetilde{C}}_{\mu, H}^{n},[v]\right)_{\Gamma}+\left(\bar{v}_{\mu, H},\left[\widetilde{C}^{n-1}-\widetilde{C}^{n}\right]\right)_{\Gamma} \\
& +d_{1} K H^{-1}\left(\left[\widetilde{C}^{n-1}-\widetilde{C}^{n}\right],[v]\right)_{\Gamma} \\
& +\left(q \tilde{c}^{n}, v\right)-\left(q^{n} c^{n}, v\right)-\left(\mathbf{u} \cdot \nabla c+\phi \frac{\partial c^{n}}{\partial t}, v\right) \\
& +\left(\phi \frac{\widetilde{C}^{n}-\widetilde{C}^{n-1}}{\Delta t_{c}}, v\right)+\left(\bar{c}_{\mu, H}^{n}-c_{\mu}^{n},[v]\right)_{\Gamma} .
\end{aligned}
$$

Subtracting (52) from (28d) and taking $v=\theta^{n}$, we adopted the skill of [4] to obtain

$$
\begin{aligned}
& \left(\phi \frac{\theta^{n}-\theta^{n-1}}{\Delta t_{c}}, \theta^{n}\right)+\left(\mathbf{D} \nabla \theta^{n}, \nabla \theta^{n}\right) \\
& +d_{1} K H^{-1}\left(\left[\theta^{n}\right],\left[\theta^{n}\right]\right)_{\Gamma}+2\left(\bar{\theta}_{\mu, H}^{n},\left[\theta^{n}\right]\right)_{\Gamma} \\
& =\left(\phi \frac{\partial c^{n}}{\partial t}+F \mathbf{u}^{n} \cdot \nabla c^{n}-\phi \frac{c^{n}-\check{c}^{n-1}}{\Delta t_{c}}, \theta^{n}\right) \\
& +\left(\left(\mathbf{u}^{n}-F \mathbf{u}^{n}\right) \cdot \nabla c^{n}, \theta^{n}\right)+\left(\phi \frac{\xi^{n}-\xi^{n-1}}{\Delta t_{c}}, \theta^{n}\right) \\
& +\left\{\left(\bar{\theta}_{\mu, H}^{n}-\bar{\theta}_{\mu, H}^{n-1},\left[\theta^{n}\right]\right)_{\Gamma}+\left(\bar{\theta}_{\mu, H}^{n},\left[\theta^{n}-\theta^{n-1}\right]\right)_{\Gamma}\right. \\
& +d_{1} K H^{-1}\left(\left[\theta^{n}-\theta^{n-1}\right],\left[\theta^{n}\right]\right)_{\Gamma} \\
& +\left(\mathbf{D} \nabla\left(\xi^{n}-\xi^{n-1}\right), \nabla \theta^{n}\right)+\left(\bar{c}_{\mu, H}^{n}-\bar{c}_{\mu, H}^{n-1},\left[\theta^{n}\right]\right)_{\Gamma} \\
& \left.+\left(c_{\mu}^{n}-\bar{c}_{\mu, H}^{n},\left[\theta^{n}\right]\right)_{\Gamma}\right\} \\
& +\left(\phi \frac{\widehat{c}^{n-1}-\check{c}^{n-1}}{\Delta t_{c}}, \theta^{n}\right)-\left(\phi \frac{\hat{\xi}^{n-1}-\check{\xi}^{n-1}}{\Delta t_{c}}, \theta^{n}\right) \\
& +\left(\phi \frac{\widehat{\theta}^{n-1}-\check{\theta}^{n-1}}{\Delta t_{c}}, \theta^{n}\right)-\left(\phi \frac{\check{\xi}^{n-1}-\xi^{n-1}}{\Delta t_{c}}, \theta^{n}\right) \\
& +\left(\phi \frac{\check{\theta}^{n-1}-\theta^{n-1}}{\Delta t_{c}}, \theta^{n}\right)+\left(q^{n}\left(\xi^{n}-\theta^{n}\right), \theta^{n}\right) .
\end{aligned}
$$

Since

$$
\begin{aligned}
\left(\bar{\theta}_{\mu, H}^{n},\left[\theta^{n}-\theta^{n-1}\right]\right)_{\Gamma}= & \left(\bar{\theta}_{\mu, H}^{n},\left[\theta^{n}\right]\right)_{\Gamma}-\left(\bar{\theta}_{\mu, H}^{n-1},\left[\theta^{n-1}\right]\right)_{\Gamma} \\
& -\left(\bar{\theta}_{\mu, H}^{n}-\bar{\theta}_{\mu, H}^{n-1},\left[\theta^{n-1}\right]\right)_{\Gamma}
\end{aligned}
$$


by summing (53) over $n$ we have

$$
\begin{aligned}
& \frac{1}{2}\left(\phi \theta^{n}, \theta^{n}\right) \\
& +\Delta t_{c}\left[\left(\mathbf{D} \nabla \theta^{n}, \nabla \theta^{n}\right)+d_{1} K H^{-1}\left(\left[\theta^{n}\right],\left[\theta^{n}\right]\right)_{\Gamma}\right. \\
& \left.+\left(\bar{\theta}_{\mu, H}^{n},\left[\theta^{n}\right]\right)_{\Gamma}\right] \\
& +\Delta t_{c} \sum_{k=1}^{n-1}\left[\left(\mathbf{D} \nabla \theta^{k}, \nabla \theta^{k}\right)+d_{1} K H^{-1}\left(\left[\theta^{k}\right],\left[\theta^{k}\right]\right)_{\Gamma}\right. \\
& \left.+2\left(\bar{\theta}_{\mu, H}^{k},\left[\theta^{k}\right]\right)_{\Gamma}\right]=\frac{1}{2}\left(\phi \theta^{0}, \theta^{0}\right) \\
& -\Delta t_{c}\left(\bar{\theta}_{\mu, H}^{0},\left[\theta^{0}\right]\right)_{\Gamma} \\
& +\Delta t_{c} \sum_{k=1}^{n}\left\{\left(\bar{\theta}_{\mu, H}^{k}-\bar{\theta}_{\mu, H}^{k-1},\left[\theta^{k}-\theta^{k-1}\right]\right)_{\Gamma}\right. \\
& +\frac{d_{1} K}{H}\left(\left[\theta^{k}-\theta^{k-1}\right],\left[\theta^{k}\right]\right)_{\Gamma} \\
& +\left(\mathbf{D} \nabla\left(\xi^{k}-\xi^{k-1}\right), \nabla \theta^{k}\right) \\
& +\left(\bar{c}_{\mu, H}^{k}-\bar{c}_{\mu, H}^{k-1},\left[\theta^{k}\right]\right)_{\Gamma} \\
& \left.+\left(c_{\mu}^{k}-\bar{c}_{\mu, H}^{k},\left[\theta^{k}\right]\right)_{\Gamma}\right\} \\
& -\sum_{k=1}^{n}\left(q^{k}\left(\xi^{k}-\theta^{k}\right), \theta^{k}\right) \Delta t_{c} \\
& +\sum_{k=1}^{n}\left(\left[\phi \frac{\partial c^{n}}{\partial t}+F \mathbf{u}^{n} \cdot \nabla c^{n}\right]-\phi \frac{c^{n}-\check{c}^{n-1}}{\Delta t_{c}}, \theta^{k}\right) \Delta t_{c} \\
& +\sum_{k=1}^{n}\left(\left(\mathbf{u}^{k}-F \mathbf{u}^{k}\right) \cdot \nabla c^{k}, \theta^{k}\right) \Delta t_{c} \\
& +\sum_{k=1}^{n}\left(\phi \frac{\xi^{k}-\xi^{k-1}}{\Delta t_{c}}, \theta^{k}\right) \Delta t_{c} \\
& +\sum_{k=1}^{n}\left(\phi \frac{\widehat{c}^{n-1}-\check{c}^{n-1}}{\Delta t_{c}}, \theta^{n}\right) \Delta t_{c} \\
& -\sum_{k=1}^{n}\left(\phi \frac{\widehat{\xi}^{k-1}-\check{\xi}^{k-1}}{\Delta t_{c}}, \theta^{k}\right) \Delta t_{c} \\
& +\sum_{k=1}^{n}\left(\phi \frac{\widehat{\theta}^{k-1}-\check{\theta}^{k-1}}{\Delta t_{c}}, \theta^{k}\right) \Delta t_{c} \\
& -\sum_{k=1}^{n}\left(\phi \frac{\check{\xi}^{k-1}-\xi^{k-1}}{\Delta t_{c}}, \theta^{k}\right) \Delta t_{c} \\
& +\sum_{k=1}^{n}\left(\phi \frac{\check{\theta}^{k-1}-\theta^{k-1}}{\Delta t_{c}}, \theta^{k}\right) \Delta t_{c} .
\end{aligned}
$$

Furthermore, noting that

$$
2\left(\bar{\theta}_{\mu, H}^{n},\left[\theta^{n}\right]\right)_{\Gamma} \leq \varepsilon\left\|\theta^{n}\right\|_{1, D}^{2}+\frac{d_{1} K}{2 \varepsilon} H^{-1}\left\|\left[\theta^{n}\right]\right\|_{L^{2}(\Gamma)}^{2},
$$

where $\|v\|_{1, D}^{2}=(\mathbf{D} \nabla v, \nabla v)$, and taking $0<\varepsilon=\sqrt{2} / 2<1$, we have

$$
\begin{aligned}
& \frac{1}{2}\left\|\theta^{n}\right\|^{2}+\frac{\Delta t_{c}}{2}\left(1-C_{0}\right)\left(\left(\mathbf{D} \nabla \theta^{n}, \nabla \theta^{n}\right)+d_{1} K H^{-1}\left\|\left[\theta^{n}\right]\right\|_{L^{2}(\Gamma)}^{2}\right) \\
& +\Delta t_{c} \sum_{k=1}^{n}\left[\frac{\Delta t_{c}}{2}\left\|\partial_{t} \theta^{k}\right\|^{2}\right. \\
& +C_{0}\left(\left(\mathbf{D} \nabla \theta^{k}, \nabla \theta^{k}\right)\right. \\
& \left.\left.+d_{1} K H^{-1}\left\|\left[\theta^{k}\right]\right\|_{L^{2}(\Gamma)}^{2}\right)\right] \\
& \leq \frac{1}{2}\left\|\theta^{0}\right\|^{2}+\Delta t_{c}\left|\left(\bar{\theta}_{\mu, H}^{0},\left[\theta^{0}\right]\right)_{\Gamma}\right| \\
& +\Delta t_{c} \sum_{k=1}^{n}\left[\left(\bar{\theta}_{\mu, H}^{k}-\bar{\theta}_{\mu, H}^{k-1},\left[\theta^{k}-\theta^{k-1}\right]\right)_{\Gamma}\right. \\
& +\frac{d_{1} K}{H}\left(\left[\theta^{k}-\theta^{k-1}\right],\left[\theta^{k}\right]\right)_{\Gamma} \\
& +\left(\mathbf{D} \nabla\left(\xi^{k}-\xi^{k-1}\right), \nabla \theta^{k}\right) \\
& +\left(\overline{\mathbf{u}}_{\mu, H}^{k}-\overline{\mathbf{u}}_{\mu, H}^{k-1},\left[\theta^{k}\right]\right)_{\Gamma} \\
& \left.+\left(\mathbf{u}_{\mu}^{k}-\overline{\mathbf{u}}_{\mu, H}^{k},\left[\theta^{k}\right]\right)_{\Gamma}\right] \\
& +\left[\sum_{k=1}^{n}\left|\left(q^{k} \theta^{k}, \theta^{k}\right)\right| \Delta t_{c}+\sum_{k=1}^{n}\left|\left(q^{k} \xi^{k}, \theta^{k}\right)\right| \Delta t_{c}\right] \\
& +\sum_{k=1}^{n}\left(\left[\phi \frac{\partial c^{k}}{\partial t}+F \mathbf{u}^{k} \cdot \nabla c^{k}\right]-\phi \frac{c^{k}-\check{c}^{k-1}}{\Delta t_{c}}, \theta^{k}\right) \Delta t_{c} \\
& +\sum_{k=1}^{n}\left(\left(\mathbf{u}^{k}-F \mathbf{u}^{k}\right) \cdot \nabla c^{k}, \theta^{k}\right) \Delta t_{c} \\
& +\sum_{k=1}^{n}\left(\phi \frac{\xi^{k}-\xi^{k-1}}{\Delta t_{c}}, \theta^{k}\right) \Delta t_{c} \\
& +\sum_{k=1}^{n}\left(\phi \frac{\widehat{c}^{k-1}-\check{c}^{k-1}}{\Delta t_{c}}, \theta^{k}\right) \Delta t_{c} \\
& +\sum_{k=1}^{n}\left|\left(\phi \frac{\hat{\xi}^{k-1}-\check{\xi}^{k-1}}{\Delta t_{c}}, \theta^{k}\right)\right| \Delta t_{c} \\
& +\sum_{k=1}^{n}\left(\phi \frac{\hat{\theta}^{k-1}-\check{\theta}^{k-1}}{\Delta t_{c}}, \theta^{k}\right) \Delta t_{c} \\
& +\sum_{k=1}^{n}\left(\phi \frac{\check{\xi}^{k-1}-\xi^{k-1}}{\Delta t_{c}}, \theta^{k}\right) \Delta t_{c} \\
& +\sum_{k=1}^{n}\left(\phi \frac{\check{\theta}^{k-1}-\theta^{k-1}}{\Delta t_{c}}, \theta^{k}\right) \Delta t_{c} .
\end{aligned}
$$


We estimate the terms on the right-hand side of (57) one by one. We denote the terms as $T_{1}, T_{2}, T_{3}, \ldots, T_{10}$ from the third term on the right-hand side of (57). We turn to analyze them one by one.

For the term $T_{1}$, similarly as that of [18], by taking

$$
\Delta t_{c} \leq \frac{d_{0}(1-\delta)^{2} C_{0}^{3} H^{2}}{16 d_{1}^{2} K^{2} C_{2}^{2}}, \quad \forall 0<\delta \ll 1,
$$

we can obtain

$$
\begin{aligned}
\left|T_{1}\right| \leq & \frac{\left(\Delta t_{c}\right)^{2}}{2} \sum_{k=1}^{n}\left\|\partial_{t} \theta^{k}\right\|^{2} \\
& +C_{0} \Delta t_{c} \sum_{k=1}^{n}\left[\left\|\theta^{k}\right\|_{1, D}^{2}+d_{0}\left\|\theta^{k}\right\|^{2}\right] \\
& +(1-\delta) C_{0} \Delta t_{c} \sum_{k=1}^{n}\left\|\theta^{k}\right\|_{1, D}^{2} \\
& +(1-\delta) C_{0} \Delta t_{c} \frac{d_{1} K}{H} \sum_{k=1}^{n}\left\|\left[\theta^{k}\right]\right\|_{L^{2}(\Gamma)}^{2} \\
& +\Delta t_{c} \sum_{k=1}^{n}\left[\frac{\Delta t_{c}}{2}\left(\left\|\theta^{k}\right\|_{1, D}^{2}+\left\|\partial_{t} \xi^{k}\right\|_{1, D}^{2}\right)\right. \\
& +C\left\|c^{k}-c^{k-1}\right\|_{1, D}^{2} \\
& +\delta C_{0} d_{1} K H^{-1}\left\|\left[\theta^{k}\right]\right\|_{L^{2}(\Gamma)}^{2} \\
& \left.+C H^{5}\left\|c^{k}\right\|_{W^{2, \infty}(\Omega)}^{2}\right] .
\end{aligned}
$$

The analyses of the terms $T_{2}, \ldots, T_{10}$ are similar to that of [4]

$$
\begin{aligned}
& \left|T_{2}\right| \leq C \Delta t_{c} \sum_{k=1}^{n}\left(\left\|\theta^{k}\right\|^{2}+\left\|\xi^{k}\right\|^{2}\right), \\
& \left|T_{3}\right| \leq C \Delta t_{c} \sum_{k=1}^{n}\left(\left\|\frac{\partial^{2} c}{\partial \tau^{2}}\right\|_{L^{2}\left(t^{k-1}, t^{k} ; L^{2}\right)} \Delta t_{c}+\left\|\theta^{k}\right\|^{2}\right), \\
& \left|T_{4}\right| \leq C \Delta t_{c}\left(\Delta t_{p}\right)^{3}\left\|\frac{\partial^{2} \mathbf{u}}{\partial t^{2}}\right\|_{L^{2}\left(t^{0}, t^{n} ; L^{2}\right)}+C \Delta t_{c} \sum_{k=1}^{n}\left\|\theta^{k}\right\|^{2}, \\
& \left|T_{5}\right| \leq C \Delta t_{c} \sum_{k=1}^{n}\left(h_{c}^{2 r+2}\|c\|_{H^{1}\left(t^{k-1}, t^{k} ; H^{r+1}\right)}^{2}+\left\|\theta^{k}\right\|^{2}\right), \\
& \left|T_{6}\right|+\left|T_{7}\right|+\left|T_{8}\right| \leq C \Delta t_{c}\left(h_{p}^{2 l+2}+h_{c}^{2 r+2}+\left(\Delta t_{c}\right)^{2}\left(\Delta t_{p}^{1}\right)^{3}\right. \\
& \left|T_{9}\right|+\left|T_{10}\right| \leq C \Delta t_{c}\left(h_{c}^{2 r+2}+\sum_{k=1}^{n}\left\|\theta^{k-1}\right\|^{4}\right)+\epsilon \Delta t_{c} \sum_{k=1}^{n}\left\|\theta^{k}\right\|_{1}^{2},
\end{aligned}
$$

Combining the above analyses, we have

$$
\begin{aligned}
\frac{1}{2}\left\|\theta^{n}\right\|^{2} & +\frac{\Delta t_{c}}{2}\left(1-C_{0}\right)\left(\left\|\theta^{n}\right\|_{1, D}^{2}+d_{1} K H^{-1}\left\|\left[\theta^{n}\right]\right\|_{L^{2}(\Gamma)}^{2}\right) \\
\leq & \frac{1}{2}\left\|\theta^{0}\right\|^{2}+\Delta t_{c}\left|\left(\bar{\theta}_{\mu, H}^{0},\left[\theta^{0}\right]\right)_{\Gamma}\right| \\
& +C \Delta t_{c} \sum_{k=1}^{n}\left[\Delta t_{c}\left(\left\|\theta^{k}\right\|_{1, D}^{2}+\left\|\partial_{t} \xi^{k}\right\|_{1, D}^{2}\right)+H^{5}\left\|\mathbf{u}^{k}\right\|_{H^{2, \infty}(\Omega)}^{2}\right] \\
& +C\left(h_{p}^{2 l+2}+h_{c}^{2 r+2}+\left(\Delta t_{c}\right)^{2}+\left(\Delta t_{p}^{1}\right)^{3}+\left(\Delta t_{p}\right)^{4}\right) .
\end{aligned}
$$

Taking $\theta^{0}=0$, applying the Gronwall Lemma and Lemmas 5 and 6 , we can derive (50).

Applying the triangle inequality, Lemmas 4 and 7, we have the following error theore.

Theorem 8. Suppose that the assumptions $(P),(Q),(I),\left(A_{p}\right)$, $\left(I_{p}\right)$ and $r \geq 1, l \geq 0$ hold. Assume that the discretization parameters obey the relations

$$
\begin{gathered}
\Delta t_{c}=o\left(h_{p}\right), \quad h_{c}^{r+1}=O\left(h_{p}\right), \\
\left(\Delta t_{p}^{1}\right)^{3 / 2}=O\left(h_{p}\right), \quad\left(\Delta t_{p}\right)^{2}=O\left(h_{p}\right) .
\end{gathered}
$$

Then for $h_{c}, h_{p}$, and $\Delta t_{c}$ sufficiently small, the errors of the approximation (28a)-(28d) for (1a)-(1d) satisfy

$$
\begin{gathered}
\max _{0 \leq n \leq N}\left\|c^{n}-C^{n}\right\| \leq C\left\{\Delta t_{c}+\left(\Delta t_{p}\right)^{2}+\left(\Delta t_{p}^{1}\right)^{3 / 2}+h_{c}^{r+1}\right. \\
\left.+h_{p}^{l+1}+H^{5 / 2}\right\}
\end{gathered}
$$

provided

$$
\Delta t_{c} \leq C_{1} H^{2}
$$

where $C_{1}$ is given by (51). Here $C$ is a positive constant dependent on $(P),(Q),(I),\left(A_{p}\right),\left(I_{p}\right),\left\|\partial^{2} c / \partial \tau^{2}\right\|,\|\partial \mathbf{u} / \partial t\|$, and $\left\|\partial^{2} \mathbf{u} / \partial t^{2}\right\|$, but independent of $h_{c}, h_{p}, \Delta t_{c}$, and $\Delta t_{p}$.

By combining Theorem 8 with (33) and (35), we obtain at once the following result.

Corollary 9. Under the assumption of Theorem 8, the errors for velocity $\mathbf{u}$, and pressure $p$ are obtained by

$$
\begin{aligned}
& \max _{0 \leq m \leq M}\left(\left\|\mathbf{u}_{m}-U_{m}\right\|_{H(\mathrm{div} ; \Omega)}+\left\|p_{m}-P_{m}\right\|\right) \\
& \quad \leq C\left\{\Delta t_{c}+\left(\Delta t_{p}\right)^{2}+\left(\Delta t_{p}^{1}\right)^{3 / 2}+h_{c}^{r+1}+h_{p}^{l+1}+H^{5 / 2}\right\} .
\end{aligned}
$$

Remark 10. As for the accuracy order of $h_{c}$ and $h_{p}$, we know that (63) and (65) are optimal for the concentration $c$, the velocity $\mathbf{u}$ and the pressure $p$. 


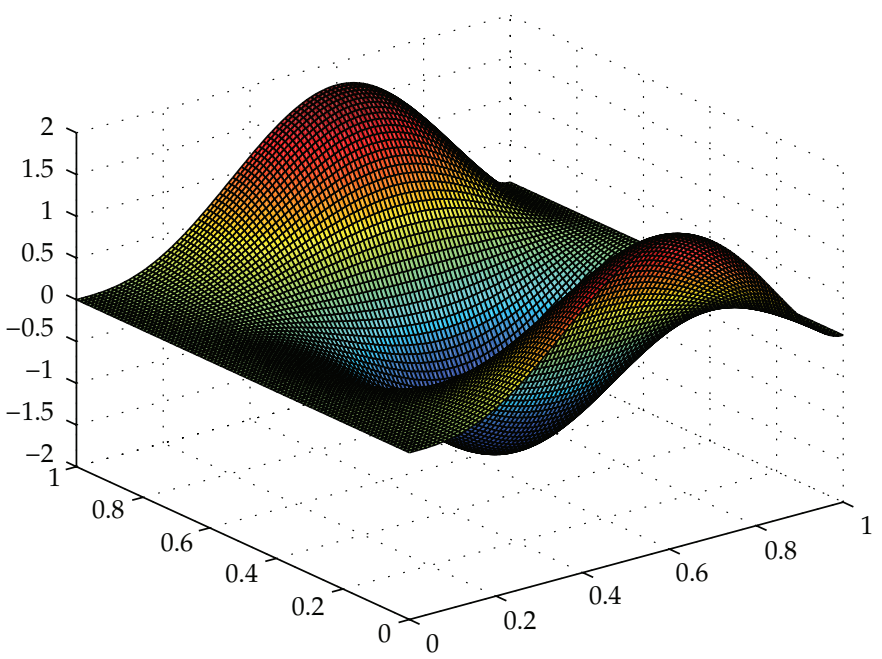

Figure 3: The exact solution $c(x, y, t)=100 t x^{3}(1-x)^{2} \cos (2 \pi y)$ at time $t=0.5$.

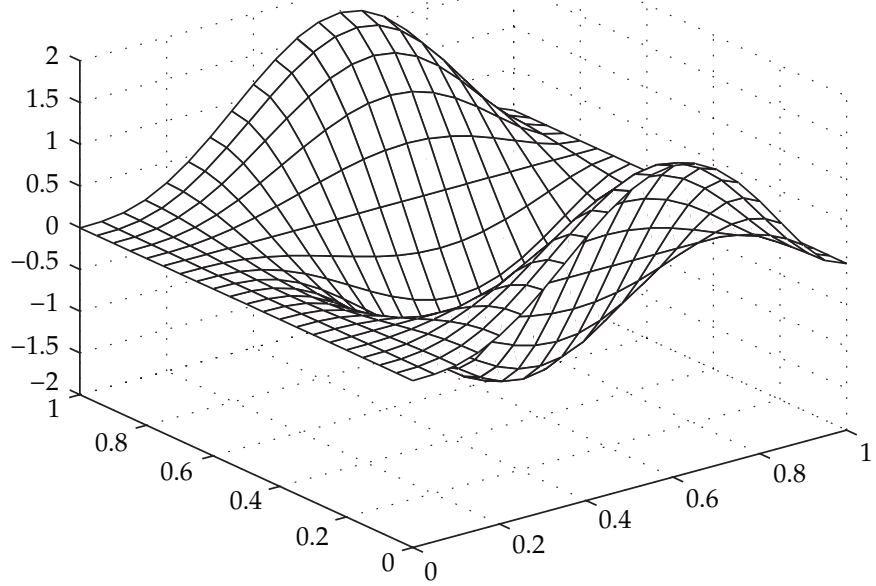

Figure 4: Approximate solution of the characteristic-DDM scheme at $t=0.5, h=1 / 40$.

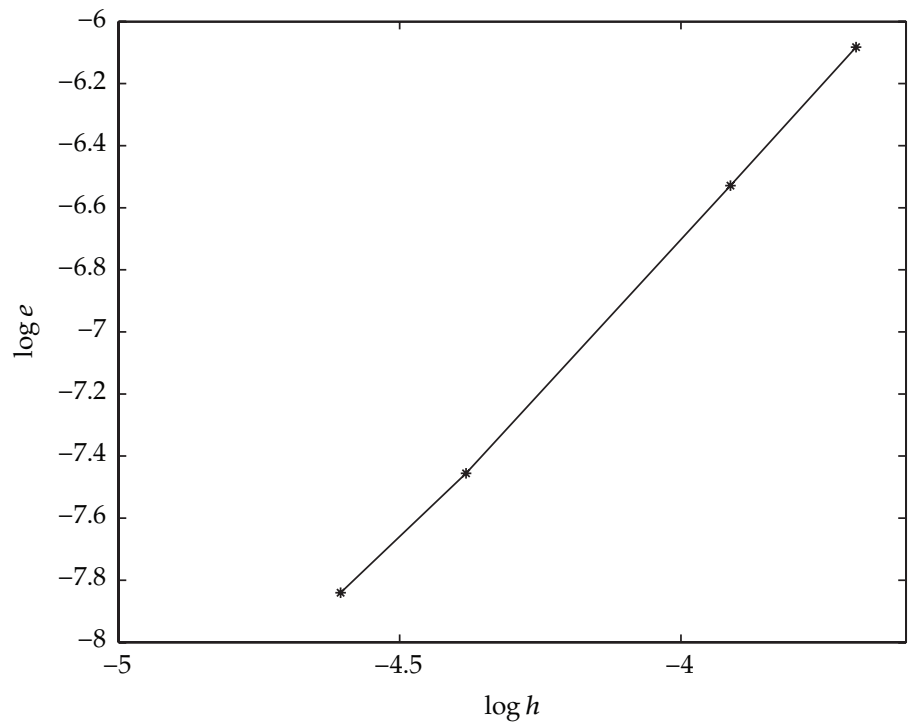

FIGURE 5: Convergence order of the characteristic-DDM scheme. 


\section{Extensions}

To get higher-order accuracy with respect to $H$, we apply the definition (22) to an extrapolation value $\widehat{C}_{H}(\mathbf{x})$ on the interdomain boundary $\Gamma$. Similar to the analysis of [18], we can present another approximate scheme for (1a)-(1d):

(a) $C^{0}=\widetilde{C}^{0}$,

(b) $A\left(C_{m} ; U_{m}, z\right)+B\left(z, P_{m}\right)=\left(\gamma\left(C_{m}\right) \nabla d, z\right), \quad \forall z \in V_{h_{p}}$,

(c) $B\left(U_{m}, w\right)=-\left(q_{m}, w\right), \quad \forall w \in W_{h_{p}}, m \geq 0$,

(d) $\left(\phi \frac{C^{n}-\check{C}^{n-1}}{\Delta t_{c}}, v\right)+\left(\mathbf{D} \nabla C^{n}, v\right)+\left(q^{n} C^{n}, v\right)$

$$
+\left(\widehat{C}_{\mu, H}^{n-1},[v]\right)_{\Gamma}+\left(\widehat{v}_{\mu, H},\left[C^{n-1}\right]\right)_{\Gamma}
$$$$
+d_{1} K H^{-1}\left(\left[C^{n-1}\right],[v]\right)_{\Gamma}=\left(q^{n} \widetilde{c}^{n}, v\right), \quad \forall v \in \mathscr{M}_{h_{c}}, n \geq 1 .
$$

Since the differences between two schemes (28a)-(28d) and (66) are the second and third term to calculate the flux on the inner-domain boundary $\Gamma$, the convergence analysis of the scheme (66) is similar to that of the scheme (28a)-(28d). For the sake of brevity, we describe the processes of analysis for (66) simply.

The proof of Theorem 15 is based on the following four lemmas. The former three lemmas are adopted from [18]. We omit the proof of Lemma 14, which is similar to that of Lemma 7 in Section 4.

Lemma 11. For sufficiently smooth function $W$, there holds estimates:

$$
\begin{gathered}
\left\|\widehat{W}_{H}-W\right\|_{L^{2}(\Gamma)} \leq \frac{2 \sqrt{2}+1}{3} \sqrt{2 H}\|\nabla W\|_{L^{2}(\Omega)}, \\
\left\|\widehat{W}_{H}-W\right\|_{L^{\infty}(\Gamma)} \leq C H^{4}\|W\|_{W^{4, \infty}(\Omega)}, \\
W(\mathbf{x})-\widehat{W}_{H}(\mathbf{x})=\frac{1}{480} H^{4} W_{v_{\Gamma}^{4}}(\mathbf{x})+o\left(H^{6}\right), \quad \forall \mathbf{x} \text { on } \Gamma,
\end{gathered}
$$

where $W_{v_{\Gamma}^{4}}$ is the fourth normal derivative of $W$ on $\Gamma$.

For functions $\psi$ with restrictions in $H^{1}\left(\Omega_{1}\right) \cup H^{1}\left(\Omega_{2}\right)$, we use the definition of the norm

$$
\|\psi \psi\|^{2}=(D \nabla \psi, \nabla \psi)+d_{1} K H^{-1}([\psi],[\psi])_{\Gamma}
$$

Lemma 12. There exists a positive constant $\widetilde{C}_{0}=1-(2 \sqrt{2} / 3)$ such that for small $H>0$,

$$
\begin{aligned}
\widetilde{C}_{0}|\|\psi\||^{2} \leq & (D \nabla \psi, \nabla \psi)+d_{1} K H^{-1}([\psi],[\psi])_{\Gamma} \\
& +2\left(\widehat{\psi}_{\mu, H},[\psi]\right)_{\Gamma}, \quad \forall v \in \mathscr{M}_{h_{c}} .
\end{aligned}
$$

Similarly as the elliptic projection (44), in order to get optimal error estimates, we introduce an elliptic projection $\widetilde{C} \in \mathscr{M}_{h_{c}}$ of the solution $u$ as follows:

$$
\begin{gathered}
(D \nabla(c-\widetilde{C}), \nabla v)+\left(\widehat{c}_{\mu, H}-\widehat{\widetilde{C}}_{\mu, H},[v]\right)_{\Gamma}+\left(\widehat{v}_{\mu, H},[c-\widetilde{C}]\right)_{\Gamma} \\
+d_{1} K H^{-1}([c-\widetilde{C}],[v])_{\Gamma}=0, \quad \forall v \in \mathscr{M}_{h_{c}} .
\end{gathered}
$$

It follows from Lemma 12 that the projection problem (70) has a unique solution for small $H$.

Let

$$
\xi^{n}=c^{n}-\widetilde{C}^{n}, \quad \theta^{n}=C^{n}-\widetilde{C}^{n} .
$$

The following lemma gives the bounds of $\xi^{n}$.

Lemma 13. There holds a priori estimates:

$$
\begin{gathered}
\|\xi\|_{L^{2}(\Omega)} \leq C\left\{h_{c}^{r+1}+H^{1 / 2}\|\eta\|_{L^{\infty}(\Omega)}\right\}, \\
\left\|\xi_{t}\right\|_{L^{2}(\Omega)} \leq C\left\{h_{c}^{r+1}+H^{1 / 2}\left\|\eta_{t}\right\|_{L^{\infty}(\Omega)}\right\} .
\end{gathered}
$$
$\theta^{n}$.

Now, we turn to derive an $L^{2}(\Omega)$-norm error estimate for

Lemma 14. For $\theta^{n}$ defined by (71), there exists the following error estimate

$$
\begin{aligned}
\max _{0 \leq n \leq N}\left\|\theta^{n}\right\|^{2} \leq C\{H & {\left[\max _{1 \leq n \leq N}\left(\left\|\eta^{n}\right\|_{L^{\infty}(\Omega)}^{2}+\left\|\eta^{n}\right\|^{2}\right)\right.} \\
& \left.+\left\|\eta_{t}\right\|_{L^{2}\left(0, T ; L^{\infty}(\Omega)\right)}^{2}+\left\|\eta_{t}\right\|_{L^{2}\left(0, T ; L^{2}(\Omega)\right)}^{2}\right] \\
+ & \left(\Delta t_{c}\right)^{2}+H^{9}+h_{p}^{2 l+2}+h_{c}^{2 r+2} \\
& \left.+\left(\Delta t_{p}^{1}\right)^{3}+\left(\Delta t_{p}\right)^{4}\right\},
\end{aligned}
$$

provided

$$
\Delta t \leq \widetilde{C}_{1} H^{2}, \quad \widetilde{C}_{1}=\frac{a_{0}(1-\delta)^{2} \widetilde{C}_{0}^{3}}{16 a_{1}^{2} K^{2} C_{2}^{2}}, \quad \forall 0<\delta \ll 1 .
$$

Theorem 15. Suppose that the assumptions $(P),(Q),(I),\left(A_{p}\right)$, $\left(I_{p}\right)$ and $r \geq 1, l \geq 0$ hold. Assume that the discretization parameters obey the relations

$$
\begin{gathered}
\Delta t_{c}=o\left(h_{p}\right), \quad h_{c}^{r+1}=O\left(h_{p}\right), \\
\left(\Delta t_{p}^{1}\right)^{3 / 2}=O\left(h_{p}\right), \quad\left(\Delta t_{p}\right)^{2}=O\left(h_{p}\right) .
\end{gathered}
$$

Then for $h_{c}, h_{p}$ and $\Delta t_{c}$ sufficiently small, the errors of the approximation (66) for (1a)-(1d) satisfy

$$
\begin{aligned}
& \max _{0 \leq n \leq N}\left\|c^{n}-C^{n}\right\| \\
& \quad \leq C\left\{\Delta t_{c}+\left(\Delta t_{p}\right)^{2}+\left(\Delta t_{p}^{1}\right)^{3 / 2}+h_{c}^{r+1}+h_{p}^{l+1}+H^{9 / 2}\right\},
\end{aligned}
$$


TABLE 1: $L^{2}$-norm error estimate of $e_{h}=c-C$ at time $t=0.5$.

\begin{tabular}{|c|c|c|c|c|c|c|}
\hline \multirow{2}{*}{ Grids } & \multirow{2}{*}{$h$} & \multirow{2}{*}{$H / h$} & \multicolumn{2}{|c|}{ Characteristic-FEM scheme } & \multicolumn{2}{|c|}{ Characteristic-DDM scheme } \\
\hline & & & $\left\|e_{h}\right\|_{L^{2}}$ & $\alpha$ & $\left\|e_{h}\right\|_{L^{2}}$ & $\alpha$ \\
\hline $50 \times 50$ & $.5000 e-01$ & $.1826 e+01$ & $.0921 e-01$ & & $.0878 e-01$ & \\
\hline $40 \times 40$ & $.2500 e-01$ & $.2091 e+01$ & $.2448 e-02$ & 1.9100 & $.2282 e-02$ & 1.9439 \\
\hline $80 \times 80$ & $.1250 e-01$ & $.2402 e+01$ & $.6245 e-03$ & 1.9708 & $.5781 e-03$ & 1.9809 \\
\hline $160 \times 160$ & $.6250 e-02$ & $.2760 e+01$ & $.1583 e-03$ & 1.9800 & $.1455 e-03$ & 1.9903 \\
\hline
\end{tabular}

provided

$$
\Delta t_{c} \leq \widetilde{C}_{1} H^{2}
$$

where $\widetilde{C}_{1}$ is given by (74). Here $C$ is a positive constant dependent on $(P),(Q),(I),\left(A_{p}\right),\left(I_{p}\right),\left\|\partial^{2} c / \partial \tau^{2}\right\|,\|\partial \mathbf{u} / \partial t\|$, $\left\|\partial^{2} \mathbf{u} / \partial t^{2}\right\|$, but independent of $h_{c}, h_{p}, \Delta t_{c}$ and $\Delta t_{p}$.

By combining Theorem 15 with (33) and (35), we obtain at once the following result.

Corollary 16. Under the assumption of Theorem 15, the errors for velocity $\mathbf{u}$ and pressure $p$ are obtained by

$$
\begin{aligned}
\max _{0 \leq m \leq M} & \left(\left\|\mathbf{u}_{m}-U_{m}\right\|_{H(\mathrm{div} ; \Omega)}+\left\|p_{m}-P_{m}\right\|\right) \\
& \leq C\left\{\Delta t_{c}+\left(\Delta t_{p}\right)^{2}+\left(\Delta t_{p}^{1}\right)^{3 / 2}+h_{c}^{r+1}+h_{p}^{l+1}+H^{9 / 2}\right\} .
\end{aligned}
$$

Remark 17. As for the accuracy order of $h_{c}$ and $h_{p}$, we know that (76) and (78) are optimal for the concentration $c$, the velocity $\mathbf{u}$ and the pressure $p$.

Remark 18. From Theorem 15, we can know that the scheme (66) has the same accuracy order as that of the scheme (28a)(28d) with respect to $\Delta t_{c}, h_{c}$ and $h_{p}$, except that has an accuracy of higher order for $H$. This shows that the scheme (66) can use larger width $H$ of middle strip domain than that of the scheme (28a)-(28d) so that the time step constraint is more weaker.

\section{Numerical Experiments}

In this section, we present some numerical experiments for the procedures described above. All computer programs below are written by Fortran 90 code and run on a Lenovo PC with Intel(R) Core i5 3.2 GHz CPU and $4 \mathrm{~GB}$ memory. The resulting linear systems of algebraic equations are solved by banded Gaussian elimination. Single precision is used for all calculations.

The main purpose of this paper is to analyze the integral mean non-overlapping DDM combined with the characteristic method for the concentration equation. There were many experimental results for the integral mean non-overlapping
DDM in $[18-20,22,23]$. For simplicity, we consider the following convection dominated diffusion equation

$$
\begin{aligned}
& \phi \frac{\partial c}{\partial t}+\mathbf{u} \cdot \nabla c-\nabla \cdot(\mathbf{D} \nabla c)+q c=f, \quad(x, t) \in \Omega \times(0, T], \\
& \frac{\partial c}{\partial \boldsymbol{v}}=0, \quad(x, t) \in \partial \Omega \times(0, T], \\
& c^{0}=0, \quad x \in \Omega, t=0,
\end{aligned}
$$

where, $\Omega=[0,1] \times[0,1], u_{1}=2+x_{1}^{2}, u_{2}=1+x_{2}^{2}, \mathbf{D}=0.05 \mathbf{I}$, $\phi=0.2, q=2$. We choose $f(x, y, t)$ suitably so that the exact solution of (79) is $c(x, y, t)=100 t x^{3}(1-x)^{2} \cos (2 \pi y)$, see Figure 3.

We consider two scenarios: (1) the characteristic implicit Galerkin scheme (Charac-teristic-FEM scheme) on uniform mesh; that is, no domain decomposition; (2) the characteristic integral mean DDM scheme (Characteristic-DDM scheme) on global uniform mesh with two equal sub-domains $\Omega_{1}=$ $(0,1 / 2) \times(0,1), \Omega_{2}=(1 / 2,1) \times(0,1)$, with the interdomain boundary $\Gamma=\{1 / 2\} \times(0,1)$. In these runs, we approximate (79) by using linear finite element with 4-node quadrilateral mesh on $20 \times 20,40 \times 40,80 \times 80$ and $160 \times 160$ grids, respectively. For each dynamic domain decomposition case, we still take $H^{5 / 2}=h^{2}$ to balance error accuracy with respect to $h, H$ and mesh ration $\Delta t=H^{5 / 2}$ in order to satisfy the conditional stability due to the explicit calculation of flux on the interface.

The following Figure 4 presents the approximate solution of the characteristic integral mean DDM scheme at time $t=$ 0.5 with space step size $h=1 / 40$.

Table 1 shows $L^{2}$-norm error estimate of $e_{h}=c-C$ at time $t=0.5$, where the error order is choosen as $\alpha=$ $\log \left(e_{h l} / e_{h l-1}\right) / \log \left(h_{l} / h_{l-1}\right)$.

Figure 5 shows the convergence order of the characteristic integral mean DDM scheme.

Remark 19. From Table 1, we see that the error estimate of the characteristic integral mean DDM scheme is better than that of the characteristic implicit Galerkin scheme. From Table 1 and Figure 5, we see that the convergence order is near 2 which is consisting with the analytical results.

\section{Acknowledgments}

The authors thank the referees for their valuable suggestions and constructive comments which led to great improvement 
of this paper. This paper was supported by the NSF of China (no. 11271231), Tian Yuan Special Foundation (no. 11126206), the Scientific Research Award Fund for Excellent Middle-Aged and Young Scientists of Shandong Province (no. BS2009NJ003) and the NSF of Shandong Province (no. ZR2010AQ019), China.

\section{References}

[1] J. Douglas, Jr., R. E. Ewing, and M. F. Wheeler, “The approximation of the pressure by a mixed method in the simulation of miscible displacement," RAIRO Analyse Numérique, vol. 17, no. 1, pp. 17-33, 1983.

[2] J. Douglas, Jr., R. E. Ewing, and M. F. Wheeler, "A timediscretization procedure for a mixed finite element approximation of miscible displacement in porous media," RAIRO Analyse Numérique, vol. 17, no. 3, pp. 249-265, 1983.

[3] R. A. Adams and J. J. F. Fournier, Sobolev Spaces, vol. 140, Academic Press, Amsterdam, The Netherlands, 2nd edition, 2003.

[4] R. E. Ewing, T. F. Russell, and M. F. Wheeler, "Convergence analysis of an approximation of miscible displacement in porous media by mixed finite elements and a modified method of characteristics," Computer Methods in Applied Mechanics and Engineering, vol. 47, no. 1-2, pp. 73-92, 1984.

[5] T. F. Russell, "Time stepping along characteristics with incomplete iteration for a Galerkin approximation of miscible displacement in porous media," SIAM Journal on Numerical Analysis, vol. 22, no. 5, pp. 970-1013, 1985.

[6] C. Johnson and V. Thomée, "Error estimates for some mixed finite element methods for parabolic type problems," RAIRO Analyse Numérique, vol. 15, no. 1, pp. 41-78, 1981.

[7] P.-A. Raviart and J. M. Thomas, "A mixed finite element method for 2nd order elliptic problems," in Mathematical Aspects of Finite Element Methods, vol. 606 of Lecture Notes in Mathematics, pp. 292-315, Springer, Berlin, Germany, 1977.

[8] J.-C. Nédélec, "Mixed finite elements in $\mathbf{R}^{3}$," Numerische Mathematik, vol. 35, no. 3, pp. 315-341, 1980.

[9] J. Douglas, Jr. and J. E. Roberts, "Global estimates for mixed methods for second order elliptic equations," Mathematics of Computation, vol. 44, no. 169, pp. 39-52, 1985.

[10] Y. R. Yuan, "Characteristic finite difference methods for positive semidefinite problem of two phase miscible flow in porous media," Systems Science and Mathematical Sciences of China, vol. 4, pp. 299-306, 1999.

[11] Y. R. Yuan, "Characteristic finite element methods for positive semidefinite problem of two phase miscible flow in three dimensions," Chinese Science Bulletin, vol. 22, pp. 2027-2032, 1996.

[12] C. N. Dawson, T. F. Russell, and M. F. Wheeler, "Some improved error estimates for the modified method of characteristics," SIAM Journal on Numerical Analysis, vol. 26, no. 6, pp. 1487$1512,1989$.

[13] M. A. Cella, T. F. Russell, I. Herrera, and R. E. Ewing, "An Eulerian-Lagrangian localized adjoint method for the advection-diffusion equations," Advances in Water Resources, vol. 13, pp. 187-206, 1990.

[14] J. H. Bramble, J. E. Pasciak, J. P. Wang, and J. Xu, "Convergence estimates for product iterative methods with applications to domain decomposition," Mathematics of Computation, vol. 57, no. 195, pp. 1-21, 1991.
[15] X.-C. Cai, "Additive Schwarz algorithms for parabolic convection-diffusion equations," Numerische Mathematik, vol. 60, no. 1, pp. 41-61, 1991.

[16] J. Xu, "Iterative methods by space decomposition and subspace correction: a unifying approach," SIAM Review, vol. 34, no. 4, pp. 581-613, 1992.

[17] X.-C. Tai, "A space decomposition method for parabolic equations," Numerical Methods for Partial Differential Equations, vol. 14, no. 1, pp. 27-46, 1998.

[18] K. Y. Ma, T. J. Sun, and D. P. Yang, "Parallel Galerkin domain decomposition procedures for parabolic equation on general domain," Numerical Methods for Partial Differential Equations, vol. 25, no. 5, pp. 1167-1194, 2009.

[19] K. Y. Ma and T. J. Sun, "Galerkin domain decomposition procedures for parabolic equations on rectangular domain," International Journal for Numerical Methods in Fluids, vol. 62, no. 4, pp. 449-472, 2010.

[20] T. J. Sun and K. Y. Ma, "Dynamic parallel Galerkin domain decomposition procedures with grid modification for parabolic equation," International Journal for Numerical Methods in Fluids, vol. 66, no. 12, pp. 1506-1529, 2011.

[21] C. N. Dawson and T. F. Dupont, "Explicit/implicit conservative Galerkin domain decomposition procedures for parabolic problems," Mathematics of Computation, vol. 58, no. 197, pp. 2134, 1992.

[22] T. J. Sun and K. Y. Ma, "Parallel Galerkin domain decomposition procedures for wave equation," Journal of Computational and Applied Mathematics, vol. 233, no. 8, pp. 1850-1865, 2010.

[23] K. Y. Ma and T. J. Sun, "Parallel Galerkin domain decomposition procedures based on the streamline diffusion method for convection-diffusion problems," Journal of Computational and Applied Mathematics, vol. 235, no. 15, pp. 4464-4479, 2011.

[24] F. Brezzi, "On the existence, uniqueness and approximation of saddle-point problems arising from Lagrangian multipliers," Revue Française d'Automatique, Informatique, Recherche Opérationnelle, vol. 8, no. R-2, pp. 129-151, 1974.

[25] P. G. Ciarlet, The Finite Element Method for Elliptic Problems, North-Holland, Amsterdam, The Netherlands, 1978.

[26] J. A. Nitsche, " $L_{\infty}$-error analysis for finite elements," in Mathematics of Finite Elements and Applications 3, J. R. Whiteman, Ed., pp. 173-186, Academic Press, London, UK, 1979.

[27] A. H. Schatz and L. B. Wahlbin, "Maximum norm estimates in the finite element method on plane polygonal domains-I," Mathematics of Computation, vol. 32, no. 141, pp. 73-109, 1978.

[28] A. H. Schatz and L. B. Wahlbin, "Maximum norm estimates in the finite element method on plane polygonal domains-II. Refinements," Mathematics of Computation, vol. 33, no. 146, pp. 465-492, 1979.

[29] S. C. Brenner and L. R. Scott, The Mathematical Theory of Finite Element Methods, vol. 15 of Texts in Applied Mathematics, Springer, New York, NY, USA, 2nd edition, 2002. 


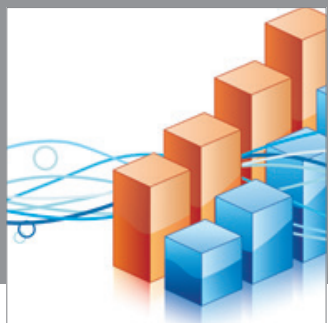

Advances in

Operations Research

mansans

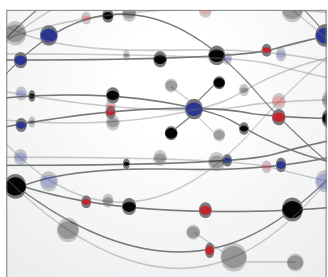

The Scientific World Journal
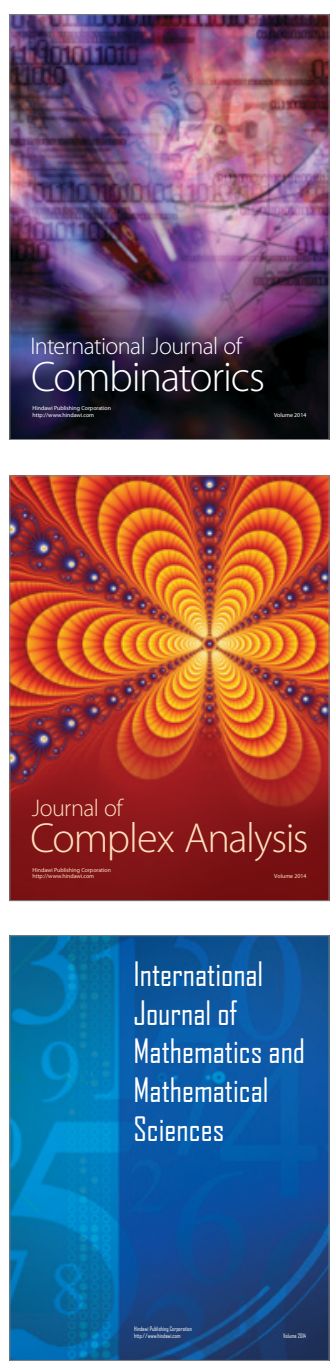
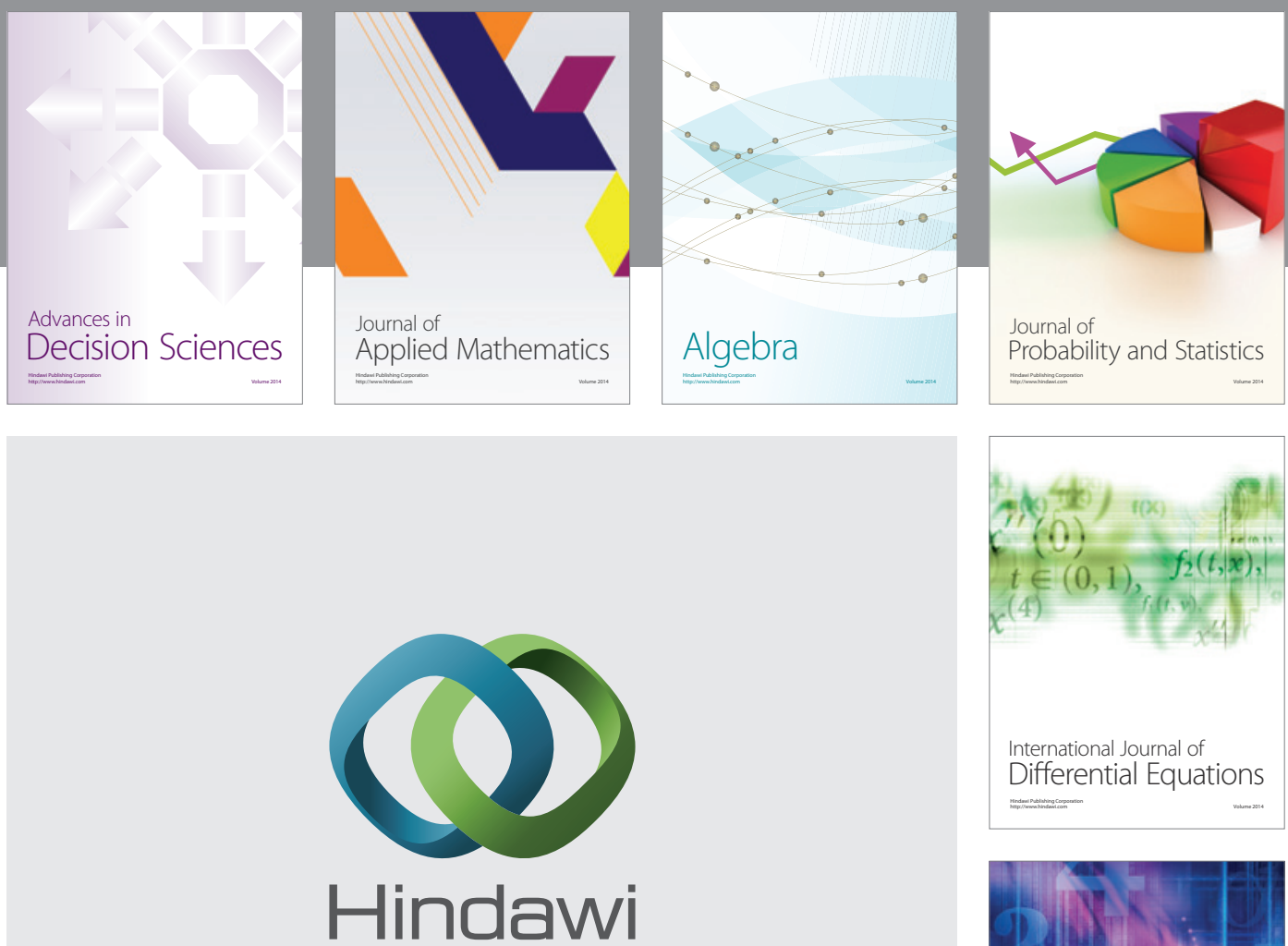

Submit your manuscripts at http://www.hindawi.com
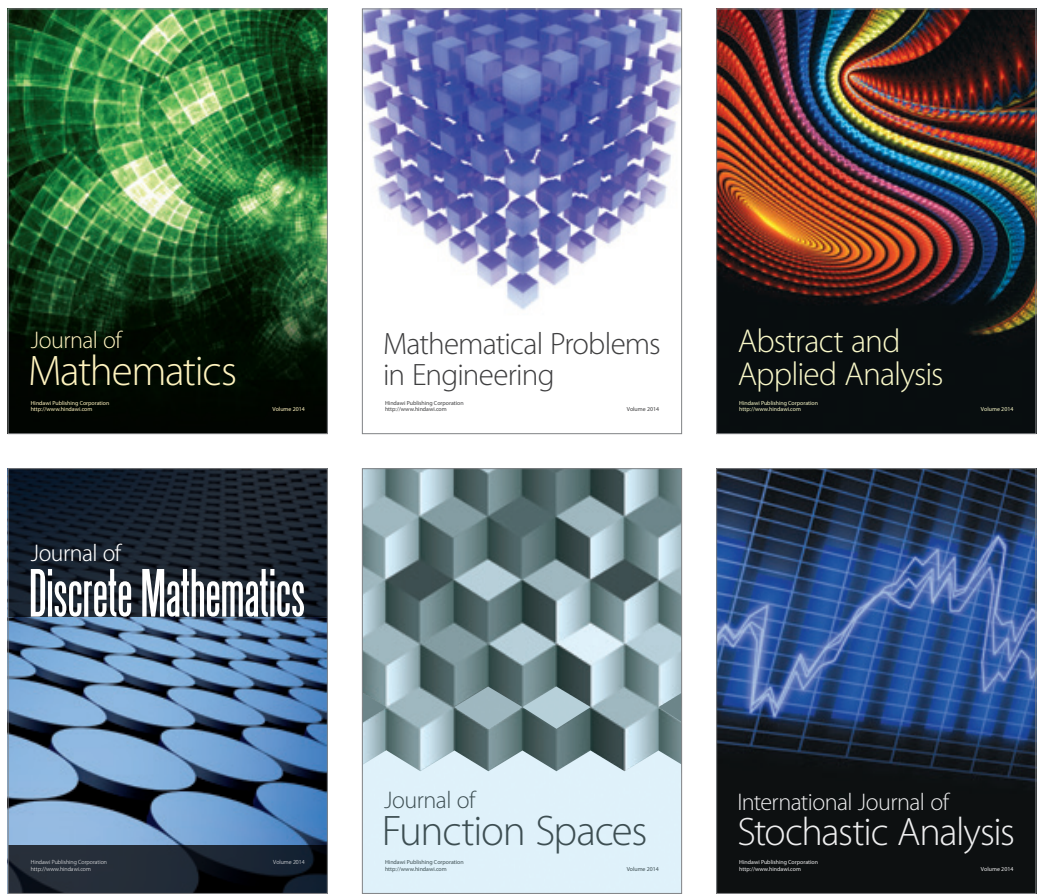

Journal of

Function Spaces

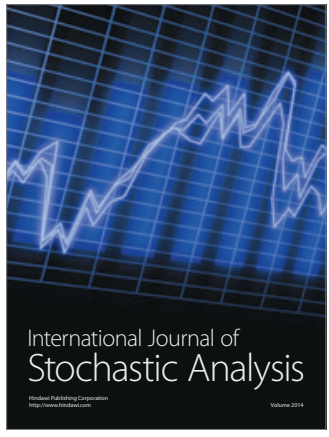

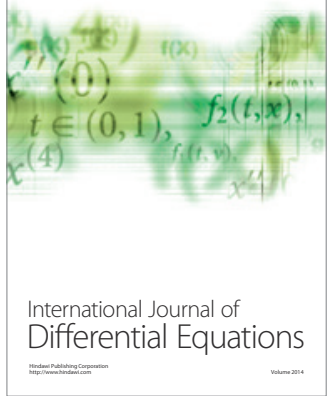
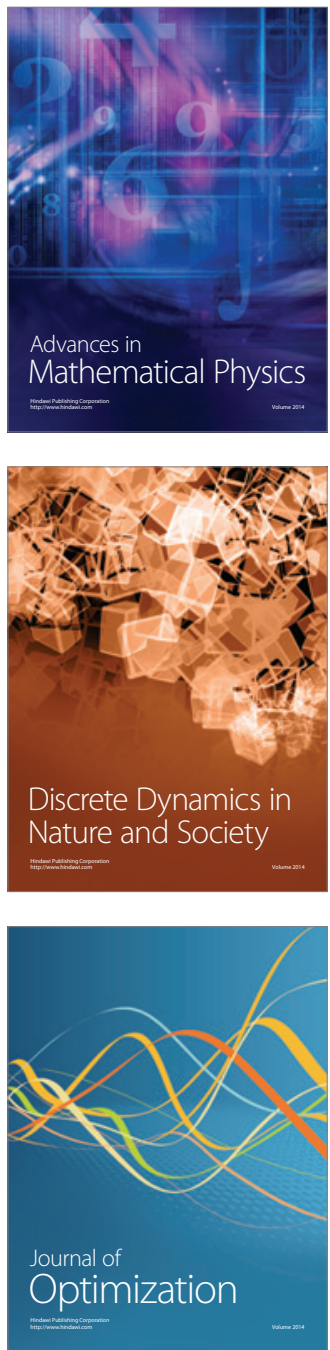